\title{
On the Resolution of Extremal and Constant Scalar Curvature Kähler Orbifolds
}

\author{
Claudio Arezzo ${ }^{1}$, Riccardo Lena ${ }^{2}$, and Lorenzo Mazzieri ${ }^{3}$ \\ ${ }^{1}$ ICTP Trieste and University of Parma, ${ }^{2}$ University of Parma, and \\ ${ }^{3}$ University of Trento, Italy \\ Correspondence to be sent to: e-mail: arezzo@ictp.it
}

Given a compact Kähler orbifold with an extremal metric, whose singularities admit a local ALE Kähler scalar-flat resolution, we prove that there exists a Kähler desingularization with an extremal metric. Moreover we study the same problem for constant scalar curvature metrics and prove some partial results.

\section{Introduction}

The aim of this paper is to give a complete answer to the following question.

Problem. Let $(M, g, \omega)$ be a compact orbifold of complex dimension $m$ endowed with an extremal metric $g$, having isolated quotient singularities at points $\left\{x_{j}\right\}_{j=1, \ldots, s}$ and orbifold groups $\Gamma_{j} \triangleleft U(m)$. Assume, in addition, that the local models for the singularities $\mathbb{C}^{m} / \Gamma_{j}$ admit scalar flat ALE Kähler resolutions $\left(X_{\Gamma_{j}}, h_{j}, \eta_{j}\right)$, then we address the following questions:

(i) Is there an extremal global resolution $(\tilde{M}, \tilde{g}, \tilde{\omega})$ of the above extremal Kähler orbifold in which suitable neighborhood of the singular points $\left\{x_{j}\right\}_{j=1, \ldots, S}$ are replaced by suitable pieces of the model spaces $\left\{X_{\Gamma_{j}}\right\}_{j=1, \ldots, S}$ for the local resolutions? 
(ii) If $g$ is a Kähler constant scalar curvature (Kcsc from now on) metric, when is the solution of the above problem Kcsc as well?

The abovementioned problem, as well as its analog when one performs blow ups of smooth points, has been the focus of extensive research, and it is now well known that the main difficulties arise in the presence of nontrivial holomorphic vector fields, so when $H^{0}(M, T M) \neq\{0\}$. It is now well understood that in the Kcsc case their presence forces the points to be in a special symplectic configuration, in order to get a positive answer to (ii). In other words, the points $\left\{x_{j}\right\}_{j=1, \ldots, S}$ must satisfy a balancing condition with respect to an $L^{2}$-orthonormal basis of the symplectic potentials $\left\{\varphi_{i}\right\}_{i=1, \ldots, d}$ of the holomorphic vector fields. On the other hand, from the works of LeBrun and Simanca [14], Arezzo et al. [4] and Székelyhidi [18], one expects the extremal problem (i) to be unobstructed. Another evidence in favor of this guess for the extremal problem comes from Tipler's solution to the above-mentioned question for surfaces with cyclic quotient singularities [19].

In the extremal case, the key difficulty lies, in fact, at the beginning, since one needs to construct Kähler potentials for the lift of the holomorphic vector fields on the local models. In the known blow up case, this had been observed in [4, Proposition 7.3], whereas Tipler observed this fact in his special case. We prove this crucial fact in complete generality in Proposition 3.3, and this paves the way to checking that the standard gluing procedure works also in this case, finally confirming in complete generality the unobstructedness of the extremal problem. This is shown in Section 5.

If $g$ is an extremal metric and $X_{S}$ its extremal vector field, we denote with $G:=\operatorname{Iso}_{0}(M, g) \cap \operatorname{Ham}(M, \omega)$ the identity component of the group of Hamiltonian isometries and with $\mathfrak{g}$ its Lie algebra. Moreover, we denote with $T \subset G$ the maximal torus whose Lie algebra $t$ contains the extremal vector field $X_{s}$ and $\tilde{T}$ its lift to the resolution. With these notations, our main result in the extremal case reads.

Theorem 1.1. Let $(M, g, \omega)$ be a compact extremal orbifold with $T$-invariant metric $g$ and singular points $\left\{x_{1}, \ldots, x_{S}\right\}$. Then there is $\bar{\varepsilon}$ such that for every $\varepsilon \in(0, \bar{\varepsilon})$ the resolution

$$
\tilde{M}:=M \sqcup_{X_{1}, \varepsilon} X_{\Gamma_{1}} \sqcup_{X_{2}, \varepsilon} \cdots \sqcup_{X_{S}, \varepsilon} X_{\Gamma_{S}}
$$

has a $\tilde{T}$-invariant extremal Kähler metric.

Once we have refined our notations and distinguished different types of singular points as below in terms of the asymptotics of the local models, we can give a more precise statement concerning the Kähler class represented by the new extremal metric 
(see Theorem 5.1). At this point, we just underline the fact that the resulting Kähler class heavily depends on the geometry of the local model.

The corresponding Kcsc problem (ii) is definitely more challenging and interesting. Passing from extremal to Kcscs, one can rely on Calabi's Theorem [7] stating that an extremal metric in a Kähler class with vanishing Futaki invariant is indeed Kcsc. Nonetheless, computing the Futaki invariant is never an easy task, and in fact, while computed in Stoppa [17], Della Vedova and Zuddas [21] and Székelyhidi [18] in the smooth blow up case is completely unknown for general resolutions. On top of this, we believe that the direct approach to the Kcsc equation is of its own interest, from the partial differential equations (PDEs) point of view.

It is clear from [1-3] that the key technical notion coming into the solution to the above-mentioned problem is the rate of convergence of the model metrics $\eta_{j}$ 's toward the euclidean metric. Indeed, a scalar flat ALE Kähler metric $\eta$ has an expansion at infinity of the form [1, Lemma 7.2]

$$
\eta= \begin{cases}\mathrm{i} \partial \bar{\partial}\left(\frac{|x|^{2}}{2}+e\left(X_{\Gamma}\right)|x|^{4-2 m}-c\left(X_{\Gamma}\right)|x|^{2-2 m}+\mathcal{O}\left(|x|^{-2 m}\right)\right) & m \geq 3 \\ \mathrm{i} \partial \bar{\partial}\left(\frac{|x|^{2}}{2}+e\left(X_{\Gamma}\right) \log (|x|)-C\left(X_{\Gamma}\right)|x|^{-2}+\mathcal{O}\left(|x|^{-4}\right)\right) & m=2 .\end{cases}
$$

The analysis required to construct Kcsc metrics with PDE's methods heavily depends on whether $e\left(X_{\Gamma}\right)$ vanishes or not. It is interesting to observe that this analytic condition does not seem to have an easy algebraic interpretation in terms of the group $\Gamma$. In fact, even in complex dimension 2, there are examples of groups that have (at least) two ALE scalar flat resolutions one with vanishing leading coefficient, and one with nonvanishing one (see Le Brun [13, Section 6, page 244; 16, Example 2, Section 6.7]). We wish to thank H.-J. Hein, C. Spotti, C. Le Brun, and I. Suvaina for many discussions about this point, and for pointing out to us these examples, which contradict our first guess. The only clear and classical fact is that if $\eta$ is Ricci flat, then $e\left(X_{\Gamma}\right)=0$ and $c\left(X_{\Gamma}\right)$ is negative. It is possible that the condition $e\left(X_{\Gamma}\right)=0$ characterizes Ricci flat metrics among scalar flat one, on minimal resolutions of the singularities.

Notation. Despite the existence of the above-mentioned examples, to keep the formulae below in a readable shape, we will denote by $e(\Gamma)$ the leading coefficient of the ALE scalar flat metric on $X_{\Gamma}$, that is, $e(\Gamma)=e\left(X_{\Gamma}\right)$, since there is no possibility of confusion among different resolutions of the same singularity. 
In terms of these coefficients, the following table collects what is known about the main problem in the Kcsc case:

\begin{tabular}{c|c|c|c}
\hline & $e\left(\Gamma_{j}\right)=0$ for all $j^{\prime} \mathrm{s}$ & $\begin{array}{c}e\left(\Gamma_{j}\right)=0 \text { for some } j^{\prime} \text { s and } \\
e\left(\Gamma_{k}\right) \neq 0 \text { for some } k\end{array}$ & $e\left(\Gamma_{k}\right) \neq 0$ for all $k$ \\
\hline$H^{0}(M, T M)=\{0\}$ & Yes ([1]) & Yes ([1]) & Yes ([1]) \\
\hline$H^{0}(M, T M) \neq\{0\}$ & $?_{1}$ & $\begin{array}{c}?_{2} \text { If dim } M>2 . \\
\text { Yes if dim (M) }(M \text { and with } \\
\text { points in special symplectic } \\
\text { position ([16]) }\end{array}$ & $\begin{array}{c}\text { Yes with points in special } \\
\text { symplectic position (easy } \\
\text { adaptation of [3]) }\end{array}$ \\
\hline
\end{tabular}

This paper gives the exact balancing conditions to answer $?_{1}$ and $?_{2}$ hence completing the solution to the above problem in terms of the asymptotics of the local models.

Theorem 1.2. Let $(M, g, \omega)$ be a Kcsc orbifold with isolated singularities. Let $\mathbf{p}=\left\{p_{1}, \ldots, p_{N}\right\} \subseteq M$ be the set of points with neighborhoods biholomorphic to a ball of $\mathbb{C}^{m} / \Gamma_{j}$ with $\Gamma_{j}$ nontrivial finite subgroup of $U(m)$ such that $\mathbb{C}^{m} / \Gamma_{j}$ admits a scalar flat ALE resolution $\left(X_{\Gamma_{j}}, h_{j}, \eta_{j}\right)$ with $e\left(X_{\Gamma_{j}}\right)=0$.

- Assume $\mathbf{q}=\left\{q_{1}, \ldots, q_{K}\right\} \subseteq M$ is the set of points with neighborhoods biholomorphic to a ball of $\mathbb{C}^{m} / \Gamma_{N+l}$ such that $\mathbb{C}^{m} / \Gamma_{N+l}$ admits a scalar flat ALE resolution $\left(Y_{\Gamma_{N+l}}, k_{l}, \theta_{l}\right)$ with $e\left(\Gamma_{N+l}\right) \neq 0$. If there exist $\mathbf{a}:=\left(a_{1}, \ldots, a_{K}\right) \in\left(\mathbb{R}^{+}\right)^{K}$ such that

$$
\begin{cases}\sum_{l=1}^{K} \frac{a_{l} e\left(\Gamma_{N+l}\right)}{\left|\left(\Gamma_{N+l}\right)\right|} \varphi_{i}\left(q_{l}\right)=0 & i=1, \ldots, d \\ \left(\frac{a_{l} e\left(\Gamma_{N+l}\right)}{\left|e\left(\Gamma_{N+l}\right)\right|} \varphi_{i}\left(q_{l}\right)\right)_{\substack{1 \leq i \leq d \\ 1 \leq l \leq K}} & \text { has full rank }\end{cases}
$$

then there exists $\varepsilon_{0}>0$ such that, for any $\varepsilon<\varepsilon_{0}$ and any $\mathbf{b}=\left(b_{1}, \ldots, b_{n}\right) \in$ $\left(\mathbb{R}^{+}\right)^{N}$, the manifold

$$
\tilde{M}:=M \sqcup_{p_{1}, \varepsilon} X_{\Gamma_{1}} \sqcup_{p_{2}, \varepsilon} \cdots \sqcup_{p_{N}, \varepsilon} X_{\Gamma_{N}} \sqcup_{q_{1}, \varepsilon} X_{\Gamma_{N+1}} \sqcup_{q_{2}, \varepsilon} \cdots \sqcup_{q_{N+K}, \varepsilon} X_{\Gamma_{N+K}},
$$

admits a Kcsc metric. 
- If $\mathbf{q}=\varnothing$ and there exists $\mathbf{b} \in \mathbb{R}_{+}^{N}$ and $\mathbf{c} \in \mathbb{R}^{N}$ such that

$$
\begin{cases}\sum_{j=1}^{N}\left(b_{j} \frac{c\left(\Gamma_{j}\right)}{\left|c\left(\Gamma_{j}\right)\right|} \Delta_{\omega} \varphi_{i}\left(p_{j}\right)+c_{j} \varphi_{i}\left(p_{j}\right)\right)=0 & i=1, \ldots, d \\ \left(\frac{c\left(\Gamma_{j}\right)}{\left|c\left(\Gamma_{j}\right)\right|}\left(b_{j} \Delta_{\omega} \varphi_{i}\left(p_{j}\right)+c_{j} \varphi_{i}\left(p_{j}\right)\right)\right)_{\substack{1 \leq i \leq d \\ 1 \leq j \leq N}} \text { has full rank }\end{cases}
$$

with

$$
c_{j}=b_{j}\left[\frac{1}{m} s_{\omega}\left(1+\frac{(m-1)^{2}}{(m+1)}\right)-\frac{c_{4, j}}{2(m-1)\left|\mathbb{S}^{2 m-1}\right|}\right]
$$

and the constants $C_{4, j}$ 's defined in formula (4.11), then there exists $\varepsilon_{0}>0$ such that for any $\varepsilon<\varepsilon_{0}$

$$
\tilde{M}:=M \sqcup_{p_{1}, \varepsilon} X_{\Gamma_{1}} \sqcup_{p_{2}, \varepsilon} \cdots \sqcup_{p_{N}, \varepsilon} X_{\Gamma_{N}}
$$

admits a Kcsc metric.

Remark 1.3. As a byproduct of our analysis, the Kähler class of the Kcsc metrics produced on $\tilde{M}$ can be specified in terms of the Kähler classes of the building blocks. More precisely, using the same notation as in the statement of Theorem 1.2, we have that

- If $\mathbf{q} \neq \emptyset$, then for every $\varepsilon<\varepsilon_{0}$ the Kcsc metrics constructed on

$$
\tilde{M}:=M \sqcup_{p_{1}, \varepsilon} X_{\Gamma_{1}} \sqcup_{p_{2}, \varepsilon} \cdots \sqcup_{p_{N}, \varepsilon} X_{\Gamma_{N}} \sqcup_{q_{1}, \varepsilon} X_{\Gamma_{N+1}} \sqcup_{q_{2}, \varepsilon} \cdots \sqcup_{q_{N+K}, \varepsilon} X_{\Gamma_{N+K}},
$$

belongs to the class

$$
\pi^{*}[\omega]+\sum_{l=1}^{K} \varepsilon^{2 m-2} \tilde{a}_{l}^{2 m-2}\left[\tilde{\theta}_{l}\right]+\sum_{j=1}^{N} \varepsilon^{2 m} b_{j}\left[\tilde{\eta}_{j}\right]
$$

where, in terms of the standard inclusions, the $\left[\tilde{\eta}_{j}\right]$ 's and the $\left[\tilde{\theta}_{l}\right]^{\prime}$ 's are respectively given by

$$
\begin{aligned}
& \mathfrak{i}_{j}^{*}\left[\tilde{\eta}_{j}\right]=\left[\eta_{j}\right] \quad \text { with } \mathfrak{i}_{j}: X_{\Gamma_{j}, R_{\varepsilon}} \hookrightarrow \tilde{M} \\
& \mathfrak{i}_{N+l}^{*}\left[\tilde{\theta}_{l}\right]=\left[\theta_{l}\right] \quad \text { with } \mathfrak{i}_{N+l}: Y_{\Gamma_{N+l}, R_{\varepsilon}} \hookrightarrow \tilde{M}
\end{aligned}
$$


whereas the coefficient $\tilde{a}_{l}$ 's are related to the ones appearing in the balancing condition (1.1). More precisely, they satisfy the estimate

$$
\begin{array}{r}
\left|\tilde{a}_{l}^{2}-\frac{\left|\Gamma_{N+l}\right| a_{l}}{4\left|\mathbb{S}^{3}\right|\left|e\left(\Gamma_{N+l}\right)\right|}\right| \leq C \varepsilon^{\gamma} \quad \text { for } m=2 \\
\left|\tilde{a}_{l}^{2 m-2}-\frac{\left|\Gamma_{N+l}\right| a_{l}}{8(m-2)(m-1)\left|\mathbb{S}^{2 m-1}\right|\left|e\left(\Gamma_{N+l}\right)\right|}\right| \leq C \varepsilon^{\gamma} \quad \text { for } m \geq 3
\end{array}
$$

for some $\gamma>0$.

- If $\mathbf{q}=\emptyset$, then for every $\varepsilon<\varepsilon_{0}$ the Kcsc metrics constructed on

$$
\tilde{M}:=M \sqcup_{p_{1}, \varepsilon} X_{\Gamma_{1}} \sqcup_{p_{2}, \varepsilon} \cdots \sqcup_{p_{N}, \varepsilon} X_{\Gamma_{N}}
$$

belongs to the class

$$
\pi^{*}[\omega]+\sum_{j=1}^{N} \varepsilon^{2 m} \tilde{b}_{j}^{2 m}\left[\tilde{\eta}_{j}\right]
$$

where the $\left[\tilde{\eta}_{j}\right]$ 's satisfy $i_{j}^{*}\left[\tilde{\eta}_{j}\right]=\left[\eta_{j}\right]$, the maps $\mathfrak{i}_{j}: X_{\Gamma_{j}, R_{\varepsilon}} \hookrightarrow \tilde{M}$ being the standard inclusions, whereas the coefficients $\tilde{b}_{j}$ are related to the ones appearing in the balancing conditions (1.2) and (1.3). More precisely, they satisfy the estimates

$$
\left|\tilde{b}_{j}^{2 m}-\frac{\left|\Gamma_{j}\right| b_{j}}{2(m-1)}\right| \leq C \varepsilon^{\gamma}
$$

for some $\gamma>0$.

In light of the above results, it would be very interesting to find a direct algebraic proof of the fact that the equations appearing in the system (1.1) and (1.2) are indeed the vanishing of the Futaki invariant of the Kähler classes we construct.

The strategy of proof follows the line of the one of [2, Theorem 1.1; 3, Theorem 1.1], and we briefly recall it here for the sake of clearness. The first step is the construction of families - depending on carefully chosen parameters - of Kcsc metrics on $M_{r_{\varepsilon}}$ (i.e., the starting orbifold $M$ with small neighborhoods of points in $\mathbf{p}$ and $\mathbf{q}$ removed) and on $X_{\Gamma_{j}, R_{\varepsilon}}$ 's and $Y_{\Gamma_{N+l}}$ 's (that are large compact sets of the local model of resolutions of points respectively of $\mathbf{p}$ and $\mathbf{q}$ ). The existence of such noncomplete Kcsc metrics follows from equations (1.1) in the first case, and (1.2) in the second one.

Taking advantage of the possibility of "moving" the families of Kcsc metrics by changing their structural parameters, it is possible to find, via an iterative procedure, 
the correct choice of parameters for which the various families match at the boundaries and produce, at once, the desingularization $\tilde{M}$ together with the Kcsc metric $\tilde{\omega}$. This step crucially needs equation (1.3), when $\mathbf{q}=\emptyset$.

As the reader can note, we assume the existence of a local model of resolution that is ALE Kähler. This is necessary if one tries to perform a gluing construction, but it is indeed a hard problem determining whether a quotient $\mathbb{C}^{m} / \Gamma$ admits a scalar flat ALE Kähler resolution. In complex dimension 2, the situation is well understood. Indeed, LeBrun [12] constructed scalar flat ALE Kähler resolutions for $\mathbb{C}^{2} / \mathbb{Z}_{k}$ with $\mathbb{Z}_{k}$ acting diagonally with same weights, Calderbank and Singer [8] provided toric (ALE Kähler) scalar flat resolutions for $\mathbb{C}^{2} / \mathbb{Z}_{k}$ for any action of $\mathbb{Z}_{k}$ and Viaclovsky and Lock [23] settled the case with $\Gamma$ nonabelian proving that there exist a scalar flat ALE Kähler metric on the minimal resolution of any $\mathbb{C}^{2} / \Gamma$. In dimension greater or equal than 3 , the class of finite subgroups of $U(m)$ acting freely on the sphere enlarges greatly, and very little is known on the existence of scalar flat ALE Kähler metrics on resolutions of the quotient singularities. It is possible to generalize to any dimension the result of [12] for group $\mathbb{Z}_{k}$ with diagonal action with same weights, and for dimension 3, a combination of the results of Joyce [11], Goto [10], Van Coevering [20], and Conlon and Hein [9] ensure the existence of Ricci-flat ALE Kähler metrics on crepant resolutions of $\mathbb{C}^{3} / \Gamma$ with $\Gamma$ a finite subgroup of SU(3).

\section{Notations and Preliminaries}

From now on, $(M, g, \omega)$ will be a Kähler orbifold with isolated singular points, with extremal metric $g$ and extremal vector field $X_{s}$. We denote with $G:=\operatorname{Iso}_{0}(M, g) \cap$ $\operatorname{Ham}(M, \omega)$ the identity component of the group of Hamiltonian isometries and with $\mathfrak{g}$ its Lie algebra. Moreover, we denote with $T \subset G$ the maximal torus whose Lie algebra $\mathfrak{t}$ contains the extremal vector field $X_{s}$. It is a standard fact (see [6]) that the action of $T$ can be linearized at fixed points, more precisely it is possible to find adapted Kähler normal coordinates in some neighborhood $U$ of fixed point $p$ such that

$$
\omega=\mathrm{i} \partial \bar{\partial}\left(\frac{|z|^{2}}{2}+\psi_{\omega}(z)\right) \quad \text { with } \quad \psi_{\omega}(z)=\mathcal{O}\left(|z|^{4}\right)
$$

and $T$ acts on $U$ as a subgroup of $U(m)$. Clearly, singular points are fixed points for the action of $G$ and hence every $\gamma \in G$ lifts to a $\tilde{\gamma} \in A u t_{0}(\tilde{M})$, so we denote with $\tilde{G}$ and $\tilde{T}$ the lifts of $G$ and of $T$ to $\tilde{M}$, respectively. 


\subsection{Singular points and local resolutions}

Then, we consider two distinguished subsets of singular points $\mathbf{p}$ and $\mathbf{q}$. We denote with $\mathbf{p}=\left\{p_{1}, \ldots, p_{N}\right\} \subseteq M$ the set of points with neighborhoods biholomorphic to a ball of $\mathbb{C}^{m} / \Gamma_{j}$ with $\Gamma_{j}$ nontrivial finite subgroup of $U(m)$ such that $\mathbb{C}^{m} / \Gamma_{j}$ admits a scalar flat ALE resolution $\left(X_{\Gamma_{j}}, h_{j}, \eta_{j}\right)$ with $e\left(\Gamma_{j}\right)=0$. Moreover, we denote with $\mathbf{q}:=\left\{q_{1}, \ldots, q_{K}\right\} \subseteq M$ be the set of points with neighborhoods biholomorphic to a ball of $\mathbb{C}^{m} / \Gamma_{N+l}$ such that $\mathbb{C}^{m} / \Gamma_{N+l}$ admits a scalar flat ALE resolution $\left(Y_{\Gamma_{N+l}}, k_{l}, \theta_{l}\right)$ with $e\left(\Gamma_{N+l}\right) \neq 0$. Whenever we will work with extremal metrics, we assume that groups $\Gamma_{N+l}$ are nontrivial. When there is no risk of confusion and no need to make the above distinction, we will just indicate by $\left(X_{\Gamma_{j}}, h_{j}, \eta_{j}\right)$ the local model, irrespective of the asymptotic of the metric.

\subsection{Eigenfunctions and eigenvalues of $\Delta_{\mathbb{S}^{2 m-1}}$}

We denote with $\mathbb{S}^{2 m-1}$ is the unit sphere of real dimension $2 m-1$, endowed with metric induced by $\left(\mathbb{C}^{m}, g_{\text {eucl }}\right)$. We also denote by $\left\{\phi_{k}\right\}_{k \in \mathbb{N}}$ a complete orthonormal system of $L^{2}\left(\mathbb{S}^{2 m-1}\right)$, generated by eigeinfunctions $\phi_{k}{ }^{\prime}$ s of $\Delta_{\mathbb{S}^{2 m-1}}$, so that, for every $k \in \mathbb{N}$,

$$
\Delta_{\mathbb{S} 2 m-1} \phi_{k}=\lambda_{k} \phi_{k}
$$

and we will indicate with $\Phi_{j}$ the generic element of the $j$ th eigenspace of $\Delta_{\mathbb{S}^{2 m-1}}$. For future convenience, we introduce the following notation, given $f \in L^{2}\left(\mathbb{S}^{2 m-1}\right)$, we denote with $f^{(k)}$ the $L^{2}\left(\mathbb{S}^{2 m-1}\right)$-projection of $f$ on the $\Lambda_{k}$-eigenspace of $\Delta_{\mathbb{S}^{2 m-1}}$ and

$$
f^{(\dagger)}:=f-f^{(0)}
$$

\subsection{The extremal equation}

We denote by $s_{\omega}$ the scalar curvature of the metric $g$ and by $\rho_{\omega}$ its Ricci form. Moreover, we denote with $\mathbf{S}_{\omega}$ the scalar curvature operator

$$
\mathbf{S}_{\omega}(\cdot): C^{\infty}(M) \longrightarrow C^{\infty}(M), \quad f \longmapsto \mathbf{S}_{\omega}(f):=s_{\omega+i \partial \bar{f}},
$$

We denote with $\mu_{\omega}: M \rightarrow \mathfrak{g}^{*}$ the Hamiltonian moment map (for a detailed account of Hamiltonian moment map, see e.g. [15]) for the action of $G$ on $M$, and we say that it is normalized if

$$
\int_{M}\left\langle\mu_{\omega}, X\right\rangle \mathrm{d} \mu_{g}=0 \quad X \in \mathfrak{g}
$$


Using an invariant scalar product on $\mathfrak{g}$ and the natural identifications, we can regard $\mu_{\omega}$ as

$$
\mu_{\omega}: M \rightarrow T M^{*}
$$

As exposed in [14], the extremal equation for $\omega$ corresponds to the prescription

$$
\bar{\partial} \partial^{\sharp} s_{\omega}=0
$$

and in terms of the Hamiltonian moment map this is equivalent to

$$
s_{\omega}=\left\langle\mu_{\omega}, X_{S}\right\rangle+\frac{1}{\operatorname{Vol}_{\omega}(M)} \int_{M} s_{\omega} \mathrm{d} \mu_{\omega} .
$$

with $X_{S}$ a holomorphic vector field on $M$.

In the sequel, we will construct families of metrics in a fixed cohomology class, so it is necessary to understand how equation (2.1) changes if we consider another Kähler metric cohomologous to $\omega$. Once we fix a Kähler class [ $\omega$ ], and we fix a Kähler form $\omega \in[\omega]$ then the extremal equation in the class $[\omega]$ is the nonlinear PDE in the unknowns $f \in C^{\infty}(M), c \in \mathbb{R}$ and $X \in H^{0}(M, T M)$

$$
\mathbf{S}_{\omega}(f)=c+\left\langle\mu_{\omega+i \partial \partial \bar{f}}, X\right\rangle+\frac{1}{\operatorname{Vol}_{\omega}(M)} \int_{M} s_{\omega} \mathrm{d} \mu_{\omega} .
$$

Remark 2.1. In Equation (2.2) an unknown constant $c$ appears, because the perturbed moment $\mu_{\omega+i \partial \bar{\partial} f}$ map is, in general, not normalized. It is hence needed this further degree of freedom to obtain the correct extremal equation.

When studying of a PDE, it is a standard procedure to consider the map, between suitable functional spaces, induced by the PDE itself. In the case of equation (2.2), the induced map

$$
\mathscr{E}: \mathcal{D} \subseteq C^{4, \alpha}(M) \times \mathbb{R} \times H^{0}(M, T M) \longrightarrow \mathbb{R}
$$

is defined as

$$
\mathscr{E}(f, c, X):=\mathbf{S}_{\omega}(f)-c-\left\langle\mu_{\omega+i \partial \bar{\partial} f}, X\right\rangle-\frac{1}{\operatorname{Vol}_{\omega}(M)} \int_{M} s_{\omega} \mathrm{d} \mu_{\omega} .
$$

and it is a matter of fact that is highly nonlinear in its variables, and the extremal metrics correspond to the triples $(f, c, X)$ such that $\mathscr{E}(f, c, X)=0$.

From now on, we will work in the $T$-invariant framework, so we indicate with $C^{k, \alpha}(M)^{T}$ the subset of $T$-invariant functions in $C^{k, \alpha}(M)$, and the definition of the map $\mathscr{E}$ 
in the $T$-invariant setting is the obvious one, that is,

$$
\mathscr{E}: \mathcal{D} \subseteq C^{4, \alpha}(M)^{T} \times \mathbb{R} \times \mathfrak{t} \longrightarrow \mathbb{R}
$$

with $\mathscr{E}$ acting in the same way as above. The following results, contained in [4], are indispensable to manipulate (2.3) in order to solve equation (2.2) in portions of the spaces we will consider in the sequel.

It is natural to look for a "Taylor expansion" for $\mathcal{E}$ at a zero, in order to study the behavior of $\mathcal{E}$ for small perturbations of an extremal metric. The first step is to understand how the moment map changes as the symplectic form moves in a fixed Kähler class, and this is done in Proposition 2.2.

Proposition 2.2. Let $(M, g, \omega)$ be a Kähler manifold with a Hamiltonian action of a Torus $T \subset G$ and $f \in C^{\infty}(M)^{T}$ such that

$$
\tilde{\omega}:=\omega+\mathrm{i} \partial \bar{\partial} f
$$

is the Kähler form of a T-invariant Kähler metric. A Hamiltonian moment map $\mu_{\tilde{\omega}}$ relative to $\tilde{\omega}$ is

$$
\left\langle\mu_{\tilde{\omega}}, X\right\rangle:=\left\langle\mu_{\omega}, X\right\rangle-\frac{1}{2} J X f
$$

With Proposition 2.3, we exploit the local structure of map $\mathscr{E}$ in a neighborhood of a zero.

Proposition 2.3. Let $(M, g, \omega)$ be a compact extremal Kähler manifold with extremal vector field $X_{S}$, with $T$-invariant metric $g$ and $\mu_{\omega}$ a normalized moment map for the action of $G$. Let $f \in C^{\infty}(M)^{T}$ such that $\omega+\mathrm{i} \partial \bar{\partial} f$ is a Kähler metric. If the triple $(f, X, c) \in$ $C^{4, \alpha}(M)^{T} \times \mathbb{R} \times \mathfrak{t}$ is sufficiently small, that is,

$$
\|f\|_{C^{4, \alpha}(M)^{T}}+|C|+\|X\|_{C^{4, \alpha}(M)^{T}}<\mathrm{C}
$$

for $C>0$ sufficiently small, then

$$
\begin{aligned}
& \mathscr{E}\left(f, c+\frac{1}{\operatorname{Vol}_{\omega}(M)} \int_{M} s_{\omega} \mathrm{d} \mu_{\omega}, X_{S}+X\right) \\
& \quad=-\frac{1}{2} \mathbb{L}_{\omega}[f]-\frac{1}{2}\left\langle\nabla s_{\omega}, \nabla f\right\rangle-\left\langle\mu_{\omega}, X\right\rangle+c+\frac{1}{2} J X f+\frac{1}{2} \mathbb{N}_{\omega}(f),
\end{aligned}
$$

where $\mathbb{L}_{\omega}$ is given by

$$
\mathbb{L}_{\omega} f=\Delta_{\omega}^{2} f+4\left\langle\rho_{\omega} \mid \mathrm{i} \partial \bar{\partial} f\right\rangle,
$$

and $\mathbb{N}_{\omega}$ is the nonlinear remainder. 
Remark 2.4. The immediate consequence of Proposition 2.3 is that if we want to solve equation (2.2) in a small neighborhood of an extremal metric then it is sufficient to solve the following equation

$$
\mathbb{L}_{\omega}[f]+\left\langle\nabla s_{\omega}, \nabla f\right\rangle+2\left\langle\mu_{\omega}, X\right\rangle=2 c+J X f+\mathbb{N}_{\omega}(f)
$$

The purpose of expanding the nonlinear map $\mathscr{E}$ is twofold: on the one hand, it is necessary to put in evidence its linearization that encodes lots of information on the solution, on the other hand, it allows us to put equation (2.2) in a form that, under some hypotheses, can be transformed in a fixed point problem and solved by means of contraction theorem. In order to translate equation (2.4) to a fixed point problem, we need to produce a right inverse for the linear operator induced by the linear part of equation (2.4). It is indeed necessary to identify the kernel of the induced linear operator and this is done in Proposition 2.5.

Proposition 2.5. Let $(M, g, \omega)$ be a compact extremal Kähler manifold and let $P_{\omega}$ : $C^{\infty}(M) \rightarrow T^{*} M \otimes T M$ be the differential operator defined by

$$
P_{\omega}[f]:=-L_{J \nabla f} J
$$

Then

$$
P_{\omega}^{*} P_{\omega}[f]=\mathbb{L}_{\omega}[f]+\left\langle\nabla s_{\omega}, \nabla f\right\rangle
$$

Moreover,

$$
\operatorname{ker}\left(P_{\omega}^{*} P_{\omega}\right) / \mathbb{R}=\left\{\left\langle\mu_{\omega}, X\right\rangle \mid X \in \mathfrak{g}\right\} .
$$

and, if we work with $T$-equivariant functions, then

$$
\operatorname{ker}\left(P_{\omega}^{*} P_{\omega}\right) / \mathbb{R}=\left\{\left\langle\mu_{\omega}, X\right\rangle \mid X \in \mathfrak{h}\right\} .
$$

where $\mathfrak{h}$ is the Lie algebra of $C_{G}(T)$, the centralizer of $T$ in $G$.

In light of the previous lemma, the extremal equation for $\omega+i \partial \bar{\partial} f$ can be rewritten as

$$
P_{\omega}^{*} P_{\omega}[f]=2 c-2\left\langle\mu_{\omega}, X\right\rangle+J X f+\mathbb{N}_{\omega}(f)
$$

Remark 2.6. As one can see, there are three unknowns in equation (2.5) that are $f \in C^{4, \alpha}(M)^{T}, c \in \mathbb{R}$, and $X \in \mathfrak{t}$ that are related one to the other. Indeed, to have a solution to the above equation, we must have that the right-hand side is $L^{2}$-orthogonal to 
12 Claudio Arezzo et al.

the kernel of $P_{\omega}^{*} P_{\omega}$ and hence the following conditions have to be satisfied

$$
C=-\frac{1}{2 \operatorname{Vol}_{\omega}(M)} \int_{M}\left[J X f+\mathbb{N}_{\omega}(f)\right] \mathrm{d} \mu_{\omega}
$$

and

$$
\int_{M}\left\langle\mu_{\omega}, X\right\rangle\left\langle\mu_{\omega}, X_{i}\right\rangle \mathrm{d} \mu_{\omega}=-\frac{1}{2} \int_{M}\left[J X f+\mathbb{N}_{\omega}(f)\right]\left\langle\mu_{\omega}, X_{i}\right\rangle \mathrm{d} \mu_{\omega} \quad i=1, \ldots, d,
$$

where $\left\{X_{1}, \ldots, X_{d}\right\}$ is basis of $\mathfrak{t}$.

\subsection{The constant scalar curvature equation}

We recall that the following expansion holds

$$
\mathbf{S}_{\omega}(f)=s_{\omega}-\frac{1}{2} \mathbb{L}_{\omega} f+\frac{1}{2} \mathbb{N}_{\omega}(f) .
$$

We recall now some well-known results on the structure of Kähler potentials of Kcsc metrics. At any point $p \in M$, there exists a holomorphic coordinate chart centered at $p$ such that $\omega$ can be written as

$$
\omega=\mathrm{i} \partial \bar{\partial}\left(\frac{|z|^{2}}{2}+\psi_{\omega}\right), \quad \text { with } \quad \psi_{\omega}=\mathcal{O}\left(|z|^{4}\right) .
$$

If in addition $s_{\omega}$ is constant, then

$$
\psi_{\omega}(z, \bar{z})=\sum_{k=0}^{+\infty} \Psi_{4+k}(z, \bar{z}),
$$

with $\Psi_{4+k}$ a real homogenous polynomials of degree $4+k$ and $\Psi_{4}$ and $\Psi_{5}$ satisfy the equations

$$
\begin{aligned}
& \Delta^{2} \Psi_{4}=-2 s_{\omega}, \\
& \Delta^{2} \Psi_{5}=0 .
\end{aligned}
$$

Let now $\Gamma \triangleleft U(m)$ be finite, a complete noncompact Kähler manifold $\left(X_{\Gamma}, h, \eta\right)$ of complex dimension $m$ is an ALE Kähler resolution of $\mathbb{C}^{m} / \Gamma$ if there exist $R>0$ and a map $\pi: X_{\Gamma} \rightarrow \mathbb{C}^{m} / \Gamma$, such that

$$
\pi: X_{\Gamma} \backslash \pi^{-1}\left(B_{R}\right) \longrightarrow\left(\mathbb{C}^{m} \backslash B_{R}\right) / \Gamma
$$

is a biholomorphism and in standard Euclidean coordinates the metric $\pi_{*} h$ satisfies the expansion

$$
\left|\frac{\partial^{\alpha}}{\partial X^{\alpha}}\left(\left(\pi_{*} h\right)_{i \bar{j}}-\frac{1}{2} \delta_{i \bar{j}}\right)\right|=\mathcal{O}\left(|X|^{-\tau-|\alpha|}\right),
$$


for some $\tau>0$ and every multindex $\alpha \in \mathbb{N}^{m}$.

If $\left(X_{\Gamma}, h, \eta\right)$ is scalar flat then for $R>0$ large enough, we have, by a result in [11], that on $X_{\Gamma} \backslash \pi^{-1}\left(B_{R}\right)$ the Kähler form can be written as

$$
\eta= \begin{cases}\mathrm{i} \partial \bar{\partial}\left(\frac{|x|^{2}}{2}+e(\Gamma)|x|^{4-2 m}-c(\Gamma)|x|^{2-2 m}+\psi_{\eta}(x)\right) & \text { with } \psi_{\eta}=\mathcal{O}\left(|x|^{-2 m}\right) \text { for } m \geq 3 \\ \mathrm{i} \partial \bar{\partial}\left(\frac{|x|^{2}}{2}+e(\Gamma) \log (|x|)-c(\Gamma)|x|^{-2}+\psi_{\eta}(x)\right) & \text { with } \psi_{\eta}=\mathcal{O}\left(|x|^{-4}\right) \text { for } m=2\end{cases}
$$

for some real constants $e(\Gamma)$ and $c(\Gamma)$ and the radial component $\psi_{\eta}^{(0)}$ in the Fourier decomposition of $\psi_{\eta}$ is such that

$$
\psi_{\eta}^{(0)}(|x|)=\mathcal{O}\left(|x|^{6-4 m}\right)
$$

If, moreover, $\Gamma \triangleleft U(m)$ is nontrivial and $e(\Gamma)=0$, the Kähler form can be written as

$$
\eta=\mathrm{i} \partial \bar{\partial}\left(\frac{|x|^{2}}{2}-c(\Gamma)|x|^{2-2 m}+\psi_{\eta}(x)\right) \quad \text { with } \psi_{\eta}=\mathcal{O}\left(|x|^{-2 m}\right),
$$

for some nonzero real constant $C(\Gamma)$ and, by [2, Proposition 2.6] the radial component $\psi_{\eta}^{(0)}$ in the Fourier decomposition of $\psi_{\eta}$ is such that

$$
\psi_{\eta}^{(0)}(|x|)=\mathcal{O}\left(|x|^{2-4 m}\right)
$$

Remark 2.7. If the ALE Kähler resolution $X_{\Gamma}$ admits a Ricci-flat (ALE Kähler) metric, then by [11, Theorem 8.2.3], the constant $c(\Gamma)$ is non-negative.

\section{Linear Analysis}

\subsection{Linear analysis on the base}

We indicate by $M_{\mathbf{p}, \mathbf{q}}:=M \backslash(\mathbf{p} \cup \mathbf{q})$, and we agree that, if $\mathbf{q}=\emptyset$, then $M_{\mathbf{p}}:=M_{\mathbf{p}, \emptyset}$ and whenever this case occurs and an object, that could be a function or a tensor, has indices relative to elements of $q$ we set these indices to 0 .

The only part of linear analysis needed to attack the extremal problem is the following fact which is proved in [4].

Proposition 3.1. Let $(M, g, \omega)$ be a compact extremal manifold with $T$-invariant metric $g$. Then, for $\delta \in(4-2 m, 5-2 m)$, the operator

$$
\mathcal{P}_{\omega, \delta}: C_{\delta}^{4, \alpha}\left(M_{\mathbf{p}, \mathbf{q}}\right)^{T} \times \mathfrak{t} \times \mathbb{R} \longrightarrow C_{\delta-4}^{0, \alpha}\left(M_{\mathbf{p}, \mathbf{q}}\right)^{T}
$$


14 Claudio Arezzo et al.

defined as

$$
\mathcal{P}_{\omega, \delta}[(f, X, c)]:=P_{\omega}^{*} P_{\omega}[f]+\left\langle\mu_{\omega}, X\right\rangle+2 c
$$

is Fredholm with

$$
\operatorname{ker}\left(\mathcal{P}_{\omega, \delta}\right) / \mathbb{R}=\mathfrak{t}
$$

From now on, the results contained in this subsection are proved in [2] and are not needed to prove Theorem 5.1 but are crucial for Theorem 1.2.

Throughout the paper, we will assume that $\operatorname{ker}\left(\mathbb{L}_{\omega}\right)$ is $(d+1)$-dimensional, and we set

$$
\operatorname{ker}\left(\mathbb{L}_{\omega}\right)=\operatorname{span}_{\mathbb{R}}\left\{\varphi_{0}, \varphi_{1}, \ldots, \varphi_{d}\right\}
$$

where $\varphi_{0} \equiv 1, d$ is a positive integer and $\varphi_{1}, \ldots, \varphi_{d}$ have zero mean and $\left\|\varphi_{i}\right\|_{L^{2}(M)}=1$, $i=1, \ldots, d$.

- We need now to recall solvability criteria for equations of the form

$$
\mathbb{L}_{\omega} u=\mu
$$

with $\mu$ a linear combination of Dirac delta's and their derivatives and elements of $\operatorname{ker}\left(\mathbb{L}_{\omega}\right)$. It was proved in [2, Proposition 3.4] that, if the following linear balancing conditions hold

$$
\begin{gathered}
\sum_{l=1}^{K} a_{l} \varphi_{i}\left(q_{l}\right)+\sum_{j=1}^{N} b_{j}\left(\Delta \varphi_{i}\right)\left(p_{j}\right)+\sum_{j=1}^{N} c_{j} \varphi_{i}\left(p_{j}\right)=0, \quad i=1, \ldots, d, \\
\sum_{l=1}^{K} a_{l}+\sum_{j=1}^{N} c_{j}=v_{\mathbf{a}, \mathbf{c}} \operatorname{Vol}_{\omega}(M),
\end{gathered}
$$

there exist a distribution $\mathbf{G}_{\mathbf{a}, \mathbf{b}, \mathbf{c}} \in \mathscr{D}^{\prime}(M)$, which satisfies the equation

$$
\mathbb{L}_{\omega}\left[\mathbf{G}_{\mathbf{a}, \mathbf{b}, \mathbf{c}}\right]+v_{\mathbf{a}, \mathbf{c}}=\sum_{l=1}^{K} a_{l} \delta_{q_{l}}+\sum_{j=1}^{N} b_{j} \Delta \delta_{p_{j}}+\sum_{j=1}^{N} c_{j} \delta_{p_{j}}, \quad \text { in } M .
$$

and we will refer to $G_{\mathbf{a}, \mathbf{b}, \mathbf{c}}$ as a multi-poles fundamental solution of $\mathbb{L}_{\omega}$. We introduce functions $G_{\Delta \Delta}(q, \cdot) \in \mathcal{C}_{\text {loc }}^{\infty}\left(M_{q}\right)$ for $q \in \mathbf{q}$ which have the expansions at $q$

$$
G_{\Delta \Delta}(q, z)= \begin{cases}|z|^{4-2 m}+\mathcal{O}\left(|z|^{6-2 m}\right) & m \geq 3 \\ \log (|z|)+C_{q}+\mathcal{O}\left(|z|^{2}\right) & m=2\end{cases}
$$


with $C_{q} \in \mathbb{R}$ and functions $G_{\Delta}(p, \cdot) \in \mathcal{C}_{\text {loc }}^{\infty}\left(M_{p}\right)$ for $p \in \mathbf{p}$ which have the expansions

$$
G_{\Delta}(p, z)= \begin{cases}|z|^{2-2 m}+|z|^{4-2 m}\left(\Phi_{2}+\Phi_{4}\right)+|z|^{5-2 m} & m \geq 3 \\ |z|^{-2}+\log (|z|)\left(\Phi_{2}+\Phi_{4}\right)+C_{p}+|z| \sum_{h=0}^{2} \Phi_{2 h+1}+\mathcal{O}\left(|z|^{2}\right) & m=2\end{cases}
$$

with $C_{p} \in \mathbb{R}$ and suitable smooth $\Gamma$-invariant functions $\Phi_{j}$ 's defined on $\mathbb{S}^{2 m-1}$ and belonging to the $j$ th eigenspace of the operator $\Delta_{\mathbb{S}^{2 m-1}}$

- Given a triple of vectors $\alpha \in \mathbb{R}^{K}$ and $\boldsymbol{\beta}, \boldsymbol{\gamma} \in \mathbb{R}^{N}$, we set, for $m \geq 3, l=1, \ldots, K$ and $j=1, \ldots, N$,

$$
\begin{aligned}
W_{\alpha}^{l} & := \begin{cases}-\frac{\alpha_{l}\left|\Gamma_{N+l}\right|}{8(m-2)(m-1)\left|\mathbb{S}^{2 m-1}\right|} G_{\Delta \Delta}\left(q_{l}, \cdot\right) & m \geq 3 \\
\frac{\alpha_{l}\left|\Gamma_{N+l \mid}\right| \mathbb{S}^{3} \mid}{4} G_{\Delta \Delta}\left(q_{l}, \cdot\right) & m=2\end{cases} \\
W_{\beta, \gamma}^{j} & = \begin{cases}\frac{\beta_{j}\left|\Gamma_{j}\right|}{2(m-1)\left|\mathbb{S}^{2 m-1}\right|} G_{\Delta}\left(p_{j}, \cdot\right)-\left(\frac{\gamma_{j}}{4}-\frac{s_{\omega}\left(m^{2}-m+2\right) \beta_{j}}{m(m+1)}\right)\left[\frac{\left|\Gamma_{j}\right|}{2(m-2)(m-1)\left|\mathbb{S}^{2 m-1}\right|} G_{\Delta \Delta}\left(p_{j}, \cdot\right)\right] & m \geq 3 \\
\beta_{j}\left[\frac{\left|\Gamma_{j}\right|}{\left|\mathbb{S}^{3}\right|} G_{\Delta}\left(p_{j}, \cdot\right)\right]+\left(\frac{\gamma_{j}}{4}-\frac{s_{\omega} \beta_{j}}{6}\right)\left[\frac{\left|\Gamma_{j}\right|}{\left|\mathbb{S}^{3}\right|} G_{\Delta \Delta}\left(p_{j}, \cdot\right)\right] & m=2\end{cases}
\end{aligned}
$$

and we define hence the deficiency spaces

$$
\mathcal{D}_{\mathbf{q}}(\boldsymbol{\alpha}):=\operatorname{span}\left\{W_{\alpha}^{l}: l=1, \ldots, K\right\} \quad \text { and } \quad \mathcal{D}_{\mathbf{p}}(\boldsymbol{\beta}, \boldsymbol{\gamma}):=\operatorname{span}\left\{W_{\boldsymbol{\beta}, \boldsymbol{\gamma}}^{j}: j=1, \ldots, N\right\} .
$$

endowed with the following norm. If $V=\sum_{l=1}^{K} V^{l} W_{\alpha}^{l} \in \mathcal{D}_{\mathbf{q}}(\boldsymbol{\alpha})$ and $U=\sum_{j=1}^{N} U^{j} W_{\boldsymbol{\beta}, \boldsymbol{\gamma}}^{j} \in \mathcal{D}_{\mathbf{p}}(\boldsymbol{\beta}, \boldsymbol{\gamma})$, we set

$$
\|V\|_{\mathcal{D}_{\mathbf{q}}(\boldsymbol{\alpha})}=\sum_{l=1}^{K}\left|V^{l}\right| \quad \text { and } \quad\|U\|_{\mathcal{D}_{\mathbf{p}}(\boldsymbol{\beta}, \boldsymbol{\gamma})}=\sum_{j=1}^{N}\left|U^{j}\right| .
$$

For the case $m=2$, we need to introduce extra deficiency spaces defined as

$$
\mathcal{E}_{\mathbf{q}}:=\operatorname{span}\left\{\chi_{q_{l}}: l=1, \ldots, K\right\} \quad \text { and } \quad \mathcal{E}_{\mathbf{p}}:=\operatorname{span}\left\{\chi_{p_{j}}: j=1, \ldots, N\right\}
$$

where the functions $\chi_{p_{1}}, \ldots, \chi_{p_{N}}, \chi_{q_{1}}, \ldots, \chi_{q_{K}}$ are smooth cutoff functions supported on small balls centered at the points $p_{1}, \ldots, p_{N}, q_{1}, \ldots, q_{K}$ and identically equal to 1 in a neighborhood of these points and they are normed in the 
16 Claudio Arezzo et al.

following way. Given two functions $X=\sum_{j=1}^{N} X^{j} \chi_{p_{j}} \in \mathcal{E}_{\mathbf{p}}$ and $Y=\sum_{l=1}^{K} Y^{l} \chi_{q_{l}} \in$ $\mathcal{E}_{\mathrm{q}}$, we set

$$
\|Y\|_{\mathcal{E}_{\mathrm{q}}}=\sum_{l=1}^{K}\left|Y^{l}\right| \quad \text { and } \quad\|X\|_{\mathcal{E}_{\mathrm{p}}}=\sum_{j=1}^{N}\left|X^{j}\right| .
$$

Given a triple of vectors $\alpha \in \mathbb{R}^{K}$ and $\boldsymbol{\beta}, \boldsymbol{\gamma} \in \mathbb{R}^{N}$, it is convenient to introduce the following matrices

$$
\begin{aligned}
\Xi_{i l}(\boldsymbol{\alpha}) & :=\alpha_{l} \frac{e\left(\Gamma_{N+l}\right)}{\left|e\left(\Gamma_{N+l}\right)\right|} \varphi_{i}\left(q_{l}\right), \quad \text { for } i=1 \ldots, d \text { and } l=1, \ldots, K, \\
\Theta_{i j}(\boldsymbol{\beta}, \boldsymbol{\gamma}) & :=\frac{c\left(\Gamma_{j}\right)}{\left|c\left(\Gamma_{j}\right)\right|}\left(\beta_{j} \Delta \varphi_{i}\left(p_{j}\right)+\gamma_{j} \varphi_{i}\left(p_{j}\right)\right), \quad \text { for } i=1 \ldots, d \text { and } j=1, \ldots, N .
\end{aligned}
$$

where $e\left(\Gamma_{N+l}\right)$ 's are defined in formula 2.7.

\subsection{Linear analysis on the model}

This subsection is crucial both for the extremal and the Kcsc case.

Let $\left(X_{\Gamma}, h, \eta\right)$ be an $A L E$ Kähler resolution and set

$$
X_{\Gamma, R_{0}}=\pi^{-1}\left(B_{R_{0}}\right),
$$

where $\pi: X_{\Gamma} \rightarrow \mathbb{C}^{m} / \Gamma$ is the canonical projection. Let $\delta \in \mathbb{R}, \alpha \in(0,1)$, the weighted Hölder space $C_{\delta}^{k, \alpha}\left(X_{\Gamma}\right)$ is the set of functions $f \in C_{\text {loc }}^{k, \alpha}\left(X_{\Gamma}\right)$ such that

$$
\|f\|_{C_{\delta}^{k, \alpha}\left(X_{\Gamma}\right)}:=\|f\|_{C^{k, \alpha}\left(X_{\Gamma, R_{0}}\right)}+\sup _{R \geq R_{0}} R^{-\delta}\|f(R \cdot)\|_{C^{k, \alpha}\left(B_{1} \backslash B_{1 / 2}\right)}<+\infty .
$$

We can now state the result [2, Proposition 4.2,4.5] that summarizes the mapping properties of $\mathbb{L}_{\eta}$ between weighted spaces on ALE Kähler spaces.

Proposition 3.2. Let $\left(X_{\Gamma}, h, \eta\right)$ a scalar flat $A L E$ Kähler manifold. If $m \geq 3$ and $\delta \in(4-2 m, 0)$, then

$$
\mathbb{L}_{\eta}^{(\delta)}: C_{\delta}^{4, \alpha}\left(X_{\Gamma}\right) \longrightarrow C_{\delta-4}^{0, \alpha}\left(X_{\Gamma}\right)
$$

is invertible. If $m=2$ and $\delta \in(0,1)$, then

$$
\mathbb{L}_{\eta}^{(\delta)}: C_{\delta}^{4, \alpha}\left(X_{\Gamma}\right) \longrightarrow C_{\delta-4}^{0, \alpha}\left(X_{\Gamma}\right)
$$


is surjective with one-dimensional kernel spanned by the constant function. If moreover $e(\Gamma)=0$ and $\Gamma \triangleleft U(m)$ is nontrivial, then for $\delta \in(2-2 m, 4-2 m)$, the operator

$$
\mathbb{L}_{\eta}^{(\delta)}: C_{\delta}^{4, \alpha}\left(X_{\Gamma}\right) \longrightarrow C_{\delta-4}^{0, \alpha}\left(X_{\Gamma}\right)
$$

has one-dimensional cokernel that is orthogonal to the space generated by functions $f \in C_{\delta-4}^{0, \alpha}\left(X_{\Gamma}\right)$ satisfying

$$
\int_{X_{\Gamma}} f \mathrm{~d} \mu_{\eta}=0
$$

Proposition 3.2 also holds trivially when restricting the domain to $T$-invariant functions, and it has the following fundamental application in producing the Kähler potentials of the lift of holomorphic vector fields.

Proposition 3.3. Let $\left(X_{\Gamma}, h, \eta\right)$ be a scalar flat ALE resolution of $\mathbb{C}^{m} / \Gamma$, then

$$
C_{U(m)}(\Gamma) \subset \mathrm{IsO}_{0}\left(X_{\Gamma}, h\right) .
$$

Proof. The standard linear action of $U(m)$ on $\mathbb{C}^{m}$ induces a natural action of $C_{U(m)}(\Gamma)$ on $\mathbb{C}^{m} / \Gamma$, and this action naturally extends to a holomorphic action on the whole $X_{\Gamma}$. It is a standard fact that every element of $C_{U(m)}(\Gamma)$ is the exponentiation of an element of $\mathfrak{u}_{\Gamma}(m) \subset \mathfrak{u}(m)$ (the algebra of skew symmetric matrices commuting with $\Gamma$ ) and hence any $\Xi \in \mathfrak{u}_{\Gamma}(m)$ gives rise to a holomorphic vector field on $\mathbb{C}^{m} / \Gamma$, that by abuse of notation we call $\Xi$, and is of the form

$$
\Xi:=\Xi_{i}^{j} z^{i} \partial_{j}
$$

To prove the result, we will show that every vector field constructed as above admits a Hamiltonian potential and hence preserves the symplectic structure $\eta$ and consequently, by the Kähler condition is also Killing. The vector field $\Xi$ is Hamiltonian for the standard symplectic structure $\omega_{\text {eucl }}$ indeed, it has the purely imaginary potential

$$
\left\langle\mu_{\mathrm{eucl}}, \Xi\right\rangle:=\Xi_{i}^{j} z^{i} \bar{z}^{j}
$$

such that

$$
\left.\bar{\partial}\left\langle\mu_{\text {eucl }}, \Xi\right\rangle=\Xi\right\lrcorner \omega_{\text {eucl }} .
$$

Denoting with $\pi: X_{\Gamma} \longrightarrow \mathbb{C}^{m} / \Gamma$, the canonical surjection we consider $\xi$ the $(0,1)$-form on $X_{\Gamma}$ defined as

$$
\xi:=\tilde{\Xi}\lrcorner\left[\eta-\pi^{*} \omega_{\text {eucl }}\right]
$$


clearly $\xi \in L^{2}\left(X_{\Gamma}, h\right)$ and moreover $\bar{\partial} \xi=0$. From [11, Theorem 8.4.1], we deduce that the first $L^{2}$-cohomology group $H_{L^{2}}^{1}\left(X_{\Gamma}, \mathbb{C}\right)$ is isomorphic to the first De Rham cohomology group $H^{1}\left(X_{\Gamma}, \mathbb{C}\right)$ and by [22, Theorem 4.1], we have that $H^{1}\left(X_{\Gamma}, \mathbb{C}\right)=\{0\}$. Moreover, by [5, Corollary 12.8$]$, we find that

$$
\xi=\bar{\partial} f
$$

with $f$ complex function in $L^{2}\left(X_{\Gamma}, h\right)$. Using the mapping properties of the Laplace operator, it is possible to obtain more information on $f$. We have, indeed, that $\xi \in C_{1-2 m}^{1, \alpha}\left(X_{\Gamma},\left(T^{*} X_{\Gamma}\right)^{(1,0)}\right)$ by construction and hence a simple computation yields $\bar{\partial}^{*} \xi \in$ $C_{-2 m}^{0, \alpha}\left(X_{\Gamma}, \mathbb{C}\right)$. Now

$$
\Delta_{\eta} f=2 \bar{\partial}^{*} \xi
$$

and using the surjectivity of the Laplace operator

$$
\Delta_{\eta}: C_{\delta}^{2, \alpha}\left(X_{\Gamma}, \mathbb{C}\right) \longrightarrow C_{\delta-2}^{0, \alpha}\left(X_{\Gamma}, \mathbb{C}\right) \quad \delta \in(3-2 m, 2-2 m)
$$

and the fact that $\Delta_{\eta}$ has no bounded kernel we conclude that $f \in C_{\delta}^{2, \alpha}\left(X_{\Gamma}, \mathbb{C}\right) \cap C_{\text {loc }}^{\infty}\left(X_{\Gamma}\right)$ with $\delta \in(3-2 m, 2-2 m)$. Now we have, by construction, that

$$
\left.\bar{\partial}\left[\pi^{*}\left\langle\mu_{\mathrm{eucl}}, \Xi\right\rangle+f\right]=\tilde{\Xi}\right\lrcorner \eta
$$

and hence

$$
\mathbb{L}_{\eta}\left[\pi^{*}\left\langle\mu_{\text {eucl }}, \Xi\right\rangle+f\right]=0
$$

Since the metric $h$ is scalar flat, $\mathbb{L}_{\eta}$ is a real operator and consequently both the real and the imaginary part of $\pi^{*}\left\langle\mu_{\text {eucl }}, \Xi\right\rangle+f$ are in the kernel of $\mathbb{L}_{\eta}$. The real part of $\pi^{*}\left\langle\mu_{\text {eucl }}, \Xi\right\rangle+f$ coincides with the real part of $f$ since $\pi^{*}\left\langle\mu_{\text {eucl }}, \Xi\right\rangle$ is purely imaginary, hence $f$ is bounded and by Proposition 3.2 it vanishes identically. We have therefore a Hamiltonian potential for the vector field $\tilde{\Xi}$ with respect to the symplectic form $\eta$. Since the flow of $\tilde{\Xi}$ preserves the complex structure and the symplectic structure, it preserves also the metric and the proposition is proved.

We point out that a consequence of Proposition 3.3 is that $\eta$ is invariant for the action of any torus in $C_{U(m)}(\Gamma)$ in particular for the action of the special torus $\tilde{T}$ we chose at the beginning. Moreover, as explained in the proof, given a vector field in $X \in \mathfrak{t}$ and denoted with $\tilde{X}$ its lift to $X_{\Gamma}$, we can always find a Hamiltonian potential $\left\langle\mu_{\eta}, \tilde{X}\right\rangle$ 
such that

$$
\left.\bar{\partial}\left\langle\mu_{\eta}, \tilde{X}\right\rangle=\tilde{X}\right\lrcorner \eta .
$$

This is a remarkable fact, indeed, a priori there could be obstructions, topological or analytical, to the existence of Hamiltonian potentials as it happens in the asymptotically conical setting. It turns out instead that, because of the special nature of the singularity $\mathbb{C}^{m} / \Gamma$, it is possible to find, on the resolutions, Hamiltonian potentials of all holomorphic vector fields coming from linear fields on $\mathbb{C}^{m} / \Gamma$.

\section{Nonlinear Analysis}

In this section, we collect all the estimates needed in the proof of Theorem 1.2. We introduce a small parameter $\varepsilon$, and we will work on the truncated manifolds (or orbifolds) $M_{r_{\varepsilon}}$ and $X_{\Gamma_{j}, R_{\varepsilon}}$ for $j=1, \ldots, N$ where we impose the relation:

$$
r_{\varepsilon}=\varepsilon^{\frac{2 m-1}{2 m+1}}=\varepsilon R_{\varepsilon}
$$

Notation. For the rest of the section, $\chi_{j}$ will denote a smooth cutoff functions identically 1 on $B_{2 r_{0}}\left(p_{j}\right)$ and identically 0 outside $B_{3 r_{0}}\left(p_{j}\right)$ and $\chi_{l}$ will denote a smooth cutoff functions identically 1 on $B_{2 r_{0}}\left(q_{l}\right)$ and identically 0 outside $B_{3 r_{0}}\left(q_{l}\right)$

\subsection{Pseudo-boundary data and biharmonic extensions}

We recall here the definition of pseudo-boundary data.

$$
\begin{aligned}
\mathcal{B}_{j} & :=C^{4, \alpha}\left(\mathbb{S}^{2 m-1} / \Gamma_{j}\right) \times C^{2, \alpha}\left(\mathbb{S}^{2 m-1} / \Gamma_{j}\right) \\
\mathcal{B}_{N+l} & :=C^{4, \alpha}\left(\mathbb{S}^{2 m-1} / \Gamma_{N+l}\right) \times C^{2, \alpha}\left(\mathbb{S}^{2 m-1} / \Gamma_{N+l}\right) \\
\mathcal{B} & :=\left(\prod_{j=1}^{N} \mathcal{B}_{j}\right) \times\left(\prod_{l=1}^{K} \mathcal{B}_{N+l}\right)
\end{aligned}
$$

for $\mathbf{q} \neq \varnothing$

$$
\mathcal{B}(\kappa, \delta):=\left\{(\mathbf{h}, \mathbf{k}) \in \mathcal{B} \mid\left\|h_{j}, k_{j}\right\|_{\mathcal{B}_{j}},\left\|h_{N+l}, k_{l}\right\|_{\mathcal{B}_{N+l}} \leq \kappa r_{\varepsilon}^{4}\right\}
$$

and for $\mathbf{q}=\emptyset$

$$
\mathcal{B}(\kappa, \delta):=\left\{(\mathbf{h}, \mathbf{k}) \in \mathcal{B}\|\| h_{j}^{(0)}, k_{j}^{(0)}\left\|_{\mathcal{B}_{j}} \leq \kappa \varepsilon^{4 m+2} r_{\varepsilon}^{-6 m+4-\delta},\right\| h_{j}^{(\dagger)}, k_{j}^{(\dagger)} \|_{\mathcal{B}_{j}} \leq \kappa \varepsilon^{2 m+4} r_{\varepsilon}^{2-4 m-\delta}\right\}
$$


Let $(h, k) \in C^{4, \alpha}\left(\mathbb{S}^{2 m-1}\right) \times C^{2, \alpha}\left(\mathbb{S}^{2 m-1}\right)$, the outer biharmonic extension of $(h, k)$ is the function $H_{h, k}^{\text {out }} \in C^{4, \alpha}\left(\mathbb{C}^{m} \backslash B_{1}\right)$ solution for the boundary value problem

$$
\begin{cases}\Delta^{2} H_{h, k}^{\text {out }}=0 & \text { on } \mathbb{C}^{m} \backslash B_{1} \\ H_{h, k}^{\text {out }}=h & \text { on } \partial B_{1} \\ \Delta H_{h, k}^{\text {out }}=k & \text { on } \partial B_{1}\end{cases}
$$

Moreover, $H_{h, k}^{\text {out }}$ has the following expansion in Fourier series

$$
H_{h, k}^{\text {out }}:= \begin{cases}\sum_{\gamma=0}^{+\infty}\left(\left(h^{(\gamma)}+\frac{k^{(\gamma)}}{4(m+\gamma-2)}\right)|w|^{2-2 m-\gamma}-\frac{k^{(\gamma)}}{4(m+\gamma-2)}|w|^{4-2 m-\gamma}\right) \phi_{\gamma} & m \geq 3 \\ h^{(0)}|w|^{-2}+\frac{k^{(0)}}{2} \log (|w|)+\sum_{\gamma=1}^{+\infty}\left(\left(h^{(\gamma)}+\frac{k^{(\gamma)}}{4 \gamma}\right)|w|^{-2-\gamma}-\frac{k^{(\gamma)}}{4 \gamma}|w|^{-\gamma}\right) \phi_{\gamma} & m=2\end{cases}
$$

Let $(\tilde{h}, \tilde{k}) \in C^{4, \alpha}\left(\mathbb{S}^{2 m-1}\right) \times C^{2, \alpha}\left(\mathbb{S}^{2 m-1}\right)$, the biharmonic extension $H_{\tilde{h}, \tilde{k}}^{\text {in }}$ on $B_{1}$ of $(\tilde{h}, \tilde{k})$ is the function $H_{\tilde{h}, \tilde{k}}^{\text {in }} \in C^{4, \alpha}\left(\bar{B}_{1}\right)$ given by the solution of the boundary value problem

$$
\begin{cases}\Delta^{2} H_{\tilde{h}, \tilde{k}}^{\text {in }}=0 & w \in B_{1} \\ H_{\tilde{h}, \tilde{k}}^{\text {in }}=\tilde{h} & w \in \partial B_{1} \\ \Delta H_{\tilde{h}, \tilde{k}}^{\text {in }}=\tilde{k} & w \in \partial B_{1}\end{cases}
$$

Moreover, the function $H_{\tilde{h}, \tilde{k}}^{\text {in }}$ has the expansion

$$
H_{\tilde{h}, \tilde{k}}^{\text {in }}(w)=\sum_{\gamma=0}^{+\infty}\left(\left(\tilde{h}^{(\gamma)}-\frac{\tilde{k}^{(\gamma)}}{4(m+\gamma)}\right)|w|^{\gamma}+\frac{\tilde{k}^{(\gamma)}}{4(m+\gamma)}|w|^{\gamma+2}\right) \phi_{\gamma} .
$$

Remark 4.1. In the sequel, we will take $\Gamma$-invariant $(h, k) \in C^{4, \alpha}\left(\mathbb{S}^{2 m-1}\right) \times C^{4, \alpha}\left(\mathbb{S}^{2 m-1}\right)$ and by [2, Remark 2.4,] we will have no terms with $\phi_{1}$ in the formula (4.3) for nontrivial $\Gamma$.

\subsection{Extremal metrics on the truncated base orbifold}

We want to construct perturbations of $\omega$ on $M_{r_{\varepsilon}}$ of the form

$$
\omega_{\mathbf{h}, \mathbf{k}}:=\omega+\mathrm{i} \partial \bar{\partial} F_{\mathbf{h}, \mathbf{k}}^{\text {out }}
$$


such that $\omega_{\mathbf{h}, \mathbf{k}}$ is extremal. Since we look for small perturbations, by Proposition 2.3, we have to find $\left(F_{\mathrm{h}, \mathrm{k}}^{\text {out }}, X^{\text {out }}, C^{\text {out }}\right) \in C^{\infty}\left(M_{r_{\varepsilon}}\right)^{T} \times \mathfrak{t} \times \mathbb{R}$ that solves equation (2.5), that is,

$$
P_{\omega}^{*} P_{\omega}\left[F_{\mathrm{h}, \mathrm{k}}^{\text {out }}\right]+2\left\langle\mu_{\omega}, X^{\text {out }}\right\rangle+2 c^{\text {out }}=-J X^{\text {out }}\left[F_{\mathrm{h}, \mathrm{k}}^{\text {out }}\right]+\mathbb{N}_{\omega}\left(F_{\mathrm{h}, \mathrm{k}}^{\text {out }}\right)
$$

We seek $F_{\mathbf{h}, \mathbf{k}}^{\text {out }}$ of the form

$$
F_{\mathrm{h}, \mathrm{k}}^{\text {out }}:=\mathbf{H}_{\mathrm{h}, \mathrm{k}}^{\text {out }}+f_{\mathrm{h}, \mathrm{k}}^{\text {out }}
$$

with

$$
\mathbf{H}_{\mathbf{h}, \mathbf{k}}^{\text {out }}:=\sum_{i=1}^{N+K} \chi_{i} H_{h_{i}, k_{i}}^{\text {out }}\left(\frac{z}{r_{\varepsilon}}\right)
$$

As usual, it is convenient to work on the punctured orbifold $M_{\mathbf{p}, \mathbf{q}}$ and to use the standard truncation/extension operators $\mathcal{E}_{r_{\varepsilon}}$, so we want to find $\left(f_{\mathrm{h}, \mathrm{k}}^{\text {out }}, X^{\text {out }}, C^{\text {out }}\right) \in$ $C_{\delta}^{4, \alpha}\left(M_{\mathbf{p}, \mathbf{q}}\right)^{T} \times \mathfrak{t} \times \mathbb{R}$ with $\delta \in(4-2 m, 5-2 m)$ such that

$$
\begin{aligned}
P_{\omega}^{*} P_{\omega}\left[f_{\mathrm{h}, \mathbf{k}}^{\text {out }}\right]+2\left\langle\mu_{\omega}, X^{\text {out }}\right\rangle+2 c^{\text {out }}= & -\mathcal{E}_{r_{\varepsilon}} P_{\omega}^{*} P_{\omega}\left[\mathbf{H}_{\mathrm{h}, \mathbf{k}}^{\text {out }}\right]-\mathcal{E}_{r_{\varepsilon}} J X^{\text {out }}\left[\mathbf{H}_{\mathrm{h}, \mathbf{k}}^{\text {out }}+f_{\mathrm{h}, \mathbf{k}}^{\text {out }}\right] \\
& +\mathcal{E}_{r_{\varepsilon}} \mathbb{N}_{\omega}\left(\mathbf{H}_{\mathbf{h}, \mathbf{k}}^{\text {out }}+f_{\mathrm{h}, \mathbf{k}}^{\text {out }}\right)
\end{aligned}
$$

Using Proposition 3.1 and Proposition 3.2, we can we rephrase equation (4.4) as a fixed point problem

$$
\left(f_{\mathbf{h}, \mathbf{k}}^{\text {out }}, X^{\text {out }}, C^{\text {out }}\right)=\mathcal{N}^{\text {out }}\left(f_{\mathbf{h}, \mathbf{k}}^{\text {out }}, X^{\text {out }}, c^{\text {out }}, \mathbf{h}, \mathbf{k}\right)
$$

with

$$
\mathcal{N}^{\text {out }}: C_{\delta}^{4, \alpha}\left(M_{\mathbf{p}, \mathbf{q}}\right)^{T} \times \mathfrak{t} \times \mathbb{R} \times \mathcal{B}(\kappa, \delta) \rightarrow C_{\delta}^{4, \alpha}\left(M_{\mathbf{p}, \mathbf{q}}\right)^{T} \times \mathfrak{t} \times \mathbb{R}
$$

nonlinear continuous operator. With computations analogous to those performed in [4], one obtains the following result.

Proposition 4.2. Let $(M, g, \omega)$ be an extremal orbifold with $T$-invariant metric $g$. Then for every $(\mathbf{h}, \mathbf{k}) \in \mathcal{B}(\kappa)$, there is $\left(f_{\mathbf{h}, \mathbf{k}}^{\text {out }}, X^{\text {out }}, c^{\text {out }}\right) \in C_{\delta}^{4, \alpha}\left(M_{\mathbf{p}, \mathbf{q}}\right)^{T} \times \mathfrak{t} \times \mathbb{R}$ with

$$
\left\|f_{\mathrm{h}, \mathbf{k}}^{\text {out }}\right\|_{C_{\delta}^{4, \alpha}\left(M_{\mathrm{p}, \mathrm{q}}\right)}+\left\|X^{\text {out }}\right\|_{C^{4, \alpha}(M)}+\left|C^{\text {out }}\right| \leq C(\kappa) r_{\varepsilon}^{2 m}
$$

such that

$$
\tilde{\omega}_{\mathbf{h}, \mathbf{k}}:=\omega+\mathrm{i} \partial \bar{\partial}\left(\mathbf{H}_{\mathbf{h}, \mathbf{k}}^{\text {out }}+f_{\mathbf{h}, \mathbf{k}}^{\text {out }}\right)
$$

is an extremal Kähler metric on $M_{r_{\varepsilon}}$ with extremal vector field $X_{S}+X^{\text {out }}$. 
It is important to note that a consequence of condition (4.5) is that around points in $\mathbf{p}$ and $\mathbf{q}$ the following estimates hold

$$
\left\|\left.f_{\mathbf{h}, \mathbf{k}}^{\text {out }}\left(r_{\varepsilon} \cdot\right)\right|_{B_{2 r_{\varepsilon}}\left(p_{j}\right)}\right\|_{C^{4, \alpha}\left(\bar{B}_{2} \backslash B_{1}\right)},\left\|\left.f_{\mathbf{h}, \mathbf{k}}^{\text {out }}\left(r_{\varepsilon} \cdot\right)\right|_{B_{2 r_{\varepsilon}}\left(q_{N+l}\right)}\right\|_{C^{4, \alpha}\left(\bar{B}_{2} \backslash B_{1}\right)} \leq \mathrm{C} r_{\varepsilon}^{4}
$$

with C positive constant depending only on $g$. This kind of estimate is indeed necessary for the success of the data matching procedure.

Another fact that deserves a word of comment is the fact that the triple $\left(f_{\mathbf{h}, \mathbf{k}}^{\text {out }}, X^{\text {out }}, c^{\text {out }}\right)$ of Proposition 4.2 depends nonlinearly upon the parameters $(\mathbf{h}, \mathbf{k})$. Indeed, being a solution of the PDE (4.4) it is immediate to see, by the structure of such equation, the nonlinear dependence of $f^{\text {out }}$ on $(\mathbf{h}, \mathbf{k})$ and then Remark 2.6 evidence the nonlinear dependence of $c^{\text {out }}$ and $X^{\text {out }}$ on $(\mathbf{h}, \mathbf{k})$ and the link between $f_{\mathbf{h}, \mathbf{k}}^{\text {out }}, X^{\text {out }}$ and $c^{\text {out }}$.

\subsection{Kcsc metrics on the truncated base orbifold}

We recall here the construction of the families of Kcsc metrics on $M_{r_{\varepsilon}}$. We want to construct $F_{\mathbf{a}, \mathbf{b}, \mathbf{c}, \mathbf{h}, \mathbf{k}}^{\text {out }} \in C^{4, \alpha}\left(M_{r_{\varepsilon}}\right)$ such that

$$
\omega_{\mathbf{a}, \mathbf{b}, \mathbf{c}, \mathbf{h}, \mathbf{k}}:=\omega+\mathrm{i} \partial \bar{\partial} F_{\mathbf{a}, \mathbf{b}, \mathbf{c}, \mathbf{h}, \mathbf{k}}^{\text {out }}
$$

is a metric on $M_{r_{\varepsilon}}$ and its scalar curvature $s_{\omega_{\mathrm{a}, \mathrm{b}, \mathrm{c}, \mathrm{h}, \mathbf{k}}}$ is a small perturbation of the scalar curvature $s_{\omega}$ of the reference Kähler metric on $M$.

The function $F_{\mathbf{a}, \mathbf{b}, \mathbf{c}, \mathbf{h}, \mathbf{k}}^{\text {out }}$ consists of blocks and takes different shapes whether $\mathbf{q}$ is empty or not

$$
F_{\mathbf{a}, \mathbf{b}, \mathbf{c}, \mathbf{h}, \mathbf{k}}^{\text {out }}:= \begin{cases}\varepsilon^{2 m-2} \mathbf{G}_{\mathbf{a}, \mathbf{0}, \mathbf{c}}+\mathbf{H}_{\mathbf{h}, \mathbf{k}}^{\text {out }}+f_{\mathbf{a}, \mathbf{0}, \mathbf{c}, \mathbf{h}, \mathbf{k}}^{\text {out }} & \mathbf{q} \neq \emptyset \\ -\varepsilon^{2 m} \mathbf{G}_{0, \mathbf{b}, \mathbf{c}}+\mathbf{P}_{\mathbf{b}, \boldsymbol{\eta}}+\mathbf{H}_{\mathbf{h}, \mathbf{k}}^{\text {out }}+f_{0, \mathbf{b}, \mathbf{c}, \mathbf{h}, \mathbf{k}}^{\text {out }} & \mathbf{q}=\emptyset\end{cases}
$$

The function $F_{\mathbf{a}, \mathbf{b}, \mathbf{c}, \mathbf{h}, \mathbf{k}}^{\text {out }}$ is made of the skeleton $\varepsilon^{2 m-2} \mathbf{G}_{\mathbf{a}, \mathbf{b}, \mathbf{c}}$, respectively, $\varepsilon^{2 m} \mathbf{G}_{\mathbf{0}, \mathbf{b}, \mathbf{c}}$ when $\mathbf{q}=\emptyset$, biharmonic extensions of pseudo-boundary data $\mathbf{H}_{\mathbf{h}, \mathbf{k}^{\prime}}^{\text {out }}$ transplanted potentials of $\eta_{j}$ 's $\mathbf{P}_{\mathbf{b}, \eta}$ when $\mathbf{q}=\emptyset$ and a "small" correction term $f_{\mathbf{a}, \mathbf{b}, \mathbf{c}, \mathbf{h}, \mathbf{k}}^{\text {out }}$ that has to be determined.

Skeleton. The skeleton is made of multi-poles fundamental solutions $\mathbf{G}_{\mathbf{a}, \mathbf{b}, \mathbf{c}}$ of $\mathbb{L}_{\omega}$ introduced in formula 3.3 that near points $p_{j}$ we have the expansion

$$
\mathbf{G}_{\mathbf{a}, \mathbf{b}, \mathbf{c}} \sim \frac{c\left(\Gamma_{j}\right) b_{j}\left|\Gamma_{j}\right|}{2\left|c\left(\Gamma_{j}\right)\right|(m-1)\left|\mathbb{S}^{2 m-1}\right|} G_{\Delta}\left(p_{j}, z\right) .
$$


and near points $q_{l}$

$$
\mathbf{G}_{\mathbf{a}, \mathbf{b}, \mathbf{c}} \sim \frac{a_{l} e\left(\Gamma_{N+l}\right)\left|\Gamma_{l}\right|}{8(m-2)(m-1)\left|e\left(\Gamma_{N+l}\right)\right|\left|\mathbb{S}^{2 m-1}\right|} G_{\Delta \Delta}\left(q_{l}, z\right)
$$

where $c\left(\Gamma_{j}\right)$ and $e\left(\Gamma_{N+l}\right)$ are introduced in formulas (2.7) and (2.8). It is then convenient, from now on, to set the following notation

$$
\begin{aligned}
A_{l} & =\left(\frac{a_{l}\left|\Gamma_{l}\right|}{8\left|e\left(\Gamma_{N+l}\right)\right|(m-2)(m-1)\left|\mathbb{S}^{2 m-1}\right|}\right)^{\frac{1}{2 m-2}} \\
B_{j} & =\left(\frac{b_{j}\left|\Gamma_{j}\right|}{2\left|C\left(\Gamma_{j}\right)\right|(m-1)\left|\mathbb{S}^{2 m-1}\right|}\right)^{\frac{1}{2 m}} \\
C_{j} & =\frac{\left|\Gamma_{j}\right|}{8(m-2)(m-1)}\left[2\left|C\left(\Gamma_{j}\right)\right| B_{j}^{2 m} \frac{(m-1)\left|\mathbb{S}^{2 m-1}\right|}{m\left|\Gamma_{j}\right|} s_{\omega}\left(1+\frac{(m-1)^{2}}{(m+1)}\right)-c_{j}\right] .
\end{aligned}
$$

Extensions of pseudo-boundary data. As in [1-3], we define for $(\mathbf{h}, \mathbf{k}) \in \mathcal{B}(\kappa, \delta)$

$$
\mathbf{H}_{\mathbf{h}, \mathbf{k}}^{\text {out }}:= \begin{cases}\sum_{j=1}^{N} \chi_{j} H_{h_{j}^{(+)}, k_{j}^{(+)}}^{\text {out }}\left(\frac{z}{r_{\varepsilon}}\right) & \mathbf{q} \neq \emptyset \\ \sum_{j=1}^{N} \chi_{j} H_{h_{j}, k_{j}^{(+)}}^{\text {out }}\left(\frac{z}{r_{\varepsilon}}\right)+\sum_{l=1}^{K} \chi_{l} H_{h_{N+l}, k_{N+l}^{(+)}}^{\text {out }}\left(\frac{z}{r_{\varepsilon}}\right) & \mathbf{q}=\emptyset\end{cases}
$$

Transplanted potentials. We need this term only when $\mathbf{q}=\emptyset$. It is the term

$$
\mathbf{P}_{\mathbf{b}, \eta}:=\sum_{j=1}^{N} B_{j}^{2} \varepsilon^{2} \chi_{j} \psi_{\eta_{j}}\left(\frac{z}{B_{j} \varepsilon}\right)
$$

with the coefficients $B_{j}$ 's defined in formula (4.6) and $\psi_{\eta_{j}}{ }^{\prime}$ s defined in formula (2.8).

Correction term. It is the term that ensures the constancy of the scalar curvature of the metric $\omega_{\mathbf{a}, \mathbf{b}, \mathbf{c}, \mathbf{h}, \mathbf{k}}$ and it is the solution on $M_{r_{\varepsilon}}$ of the equations in the unknown $f$

$$
\begin{aligned}
\mathbb{L}_{\omega}[f]= & \left(2 s_{\omega}-\varepsilon^{2 m-2} v_{\mathbf{0}, \mathbf{c}}-2 s_{\omega_{0, \mathbf{b}, \mathbf{c}, \mathbf{h}, \mathbf{k}}}\right)-\mathbb{L}_{\omega}\left[\mathbf{P}_{\mathbf{b}, \boldsymbol{\eta}}\right]-\mathbb{L}_{\omega}\left[\mathbf{H}_{\mathbf{h}, \mathbf{k}}^{\text {out }}\right] \\
& +\mathbb{N}_{\omega}\left(-\varepsilon^{2 m-2} \mathbf{G}_{\mathbf{0}, \mathbf{b}, \mathbf{c}}+\mathbf{P}_{\mathbf{b}, \boldsymbol{\eta}}+\mathbf{H}_{\mathbf{h}, \mathbf{k}}^{\text {out }}+f\right),
\end{aligned}
$$


if $\mathbf{q}=\emptyset$ and

$$
\begin{aligned}
\mathbb{L}_{\omega}[f]= & \left(2 s_{\omega}+\varepsilon^{2 m-2} v_{\mathbf{a}, \mathbf{c}}-2 s_{\omega_{\mathbf{a}, 0, \mathbf{c}, \mathbf{h}, \mathbf{k}}}\right)-\mathbb{L}_{\omega}\left[\mathbf{H}_{\mathbf{h}, \mathbf{k}}^{\text {out }}\right] \\
& +\mathbb{N}_{\omega}\left(+\varepsilon^{2 m-2} \mathbf{G}_{\mathbf{a}, \mathbf{0}, \mathbf{c}}+\mathbf{H}_{\mathbf{h}, \mathbf{k}}^{\text {out }}+f\right) .
\end{aligned}
$$

if $\mathbf{q} \neq \emptyset$.

It is a function $f_{\mathbf{a}, \mathbf{b}, \mathbf{c}, \mathbf{h}, \mathbf{k}}^{\text {out }} \in C_{\delta}^{4, \alpha}\left(M_{\mathbf{p}, \mathbf{q}}\right) \oplus \mathcal{D}_{\mathbf{p}}(\mathbf{b}, \mathbf{c}) \oplus \mathcal{D}_{\mathbf{q}}(\mathbf{a})$ if $m \geq 3$ and $f_{\mathbf{a}, \mathbf{b}, \mathbf{c}, \mathbf{h}, \mathbf{k}}^{\text {out }} \in C_{\delta}^{4, \alpha}\left(M_{\mathbf{p}, \mathbf{q}}\right) \oplus \mathcal{E}_{\mathbf{p}} \oplus \mathcal{D}_{\mathbf{p}}(\mathbf{b}, \mathbf{c}) \oplus \mathcal{E}_{\mathbf{q}} \oplus \mathcal{D}_{\mathbf{q}}(\mathbf{a})$ if $m=2$, where the spaces $C_{\delta}^{4, \alpha}\left(M_{\mathbf{p}, \mathbf{q}}\right) \oplus \mathcal{D}_{\mathbf{p}}(\mathbf{b}, \mathbf{c}) \oplus \mathcal{D}_{\mathbf{q}}(\mathbf{a})$ and $C_{\delta}^{4, \alpha}\left(M_{\mathbf{p}, \mathbf{q}}\right) \oplus \mathcal{E}_{\mathbf{p}} \oplus \mathcal{D}_{\mathbf{p}}(\mathbf{b}, \mathbf{c}) \oplus \mathcal{E}_{\mathbf{q}} \oplus \mathcal{D}_{\mathbf{q}}(\mathbf{a})$ are defined in formulas (3.1) and (3.2).

Notation. For the rest of the paper, we will denote with $\mathrm{C}$ a positive constant, that can vary from line to line, depending only on $\omega, \eta_{j}$ 's and $\theta_{N+l}$ 's.

We can now state the main propositions for the base space. The first deals with the case $q \neq \emptyset$, and its proof is an easy adaptation of [3, proof of Proposition 5.1]

Proposition 4.3. Let $(M, g, \omega)$ a Kcsc orbifold with isolated singularities, and let $\mathbf{p}$ be the set of singular points with nontrivial orbifold group that admit a scalar flat ALE resolution with $e(\Gamma)=0$ and $\mathbf{q}$ a nonempty set of points admitting scalar flat ALE resolutions with $e(\Gamma) \neq 0$.

- Assume exist $\mathbf{a} \in\left(\mathbb{R}^{+}\right)^{K}$ such that

$$
\begin{cases}\sum_{l=1}^{K} a_{l} \frac{e\left(\Gamma_{N+l}\right)}{\left|e\left(\Gamma_{N+l}\right)\right|} \varphi_{i}\left(q_{l}\right)=0 & i=1, \ldots, d \\ (\Xi(\mathbf{a}))_{\substack{1 \leq i \leq d \\ 1 \leq l \leq K}} & \text { has full rank, }\end{cases}
$$

where $(\Xi(\mathbf{a}))_{\substack{1 \leq i \leq d \\ 1 \leq l \leq K}}$ is the matrix introduced in Section 3 formula (3.3). Let $\mathbf{G}_{\mathbf{a}, \mathbf{b}, \mathbf{c}}$ be the multi-poles solution of $\mathbb{L}_{\omega}$ introduced in Section 3 Remark 3.3.

- Let $\delta \in(4-2 m, 5-2 m)$. Given any $(\mathbf{h}, \mathbf{k}) \in \mathcal{B}(\kappa, \delta)$, where $\mathcal{B}(\kappa, \delta)$ is the space defined in formula (4.1), let $\mathbf{H}_{\mathrm{h}, \mathrm{k}}^{\text {out }}$ be the function defined in formula (4.7).

$$
\mathbf{H}_{\mathbf{h}, \mathbf{k}}^{\text {out }}:=\sum_{j=1}^{N} \chi_{j} H_{h_{j}, k_{j}^{(\dagger)}}^{\text {out }}\left(\frac{z}{r_{\varepsilon}}\right)+\sum_{l=1}^{K} \chi_{l} H_{h_{N+l}, k_{N+l}^{(+)}}^{\text {out }}\left(\frac{z}{r_{\varepsilon}}\right)
$$


Then for every $|\mathbf{c}| \leq r_{\varepsilon}^{4}$, there is $\overline{\mathbf{a}} \in\left(\mathbb{R}^{+}\right)^{K}$ such that

$$
|\mathbf{a}-\overline{\mathbf{a}}| \leq \mathrm{Cr}_{\varepsilon}^{2 m} \varepsilon^{2-2 m}
$$

and $f_{\mathbf{a}, \mathbf{0}, \mathbf{c}, \mathbf{h}, \mathbf{k}}^{\text {out }} \in C_{\delta}^{4, \alpha}\left(M_{\mathbf{p}, \mathbf{q}}\right) \oplus \mathcal{D}_{\mathbf{q}}(\overline{\mathbf{a}})$ if $m \geq 3$ and $f_{\mathbf{a}, \mathbf{0}, \mathbf{c}, \mathbf{h}, \mathbf{k}}^{\text {out }} \in C_{\delta}^{4, \alpha}\left(M_{\mathbf{p}, \mathbf{q}}\right) \oplus \mathcal{E}_{\mathbf{p}} \oplus \mathcal{E}_{\mathbf{q}} \oplus \mathcal{D}_{\mathbf{q}}(\overline{\mathbf{a}})$ if $m=2$ such that

$$
\omega_{\mathbf{a}, \mathbf{0}, \mathbf{c}, \mathbf{h}, \mathbf{k}}=\omega+i \partial \bar{\partial}\left(\mathbf{G}_{\overline{\mathbf{a}}, 0, \mathbf{c}}+\mathbf{H}_{\mathbf{h}, \mathbf{k}}^{\text {out }}+f_{\mathbf{a}, \mathbf{0}, \mathbf{c}, \mathbf{h}, \mathbf{k}}^{\text {out }}\right)
$$

is a Kcsc metric on $M_{r_{\varepsilon}}$ and the following estimates hold.

$$
\begin{array}{r}
\left\|f_{\mathbf{a}, \mathbf{0}, \mathbf{c}, \mathbf{h}, \mathbf{k}}^{\text {out }}\right\|_{C_{\delta}^{4, \alpha}\left(M_{\mathbf{p}, \mathbf{q}}\right) \oplus \mathcal{D}_{\mathbf{q}}(\overline{\mathbf{a}})} \leq \mathrm{Cr}_{\varepsilon}^{2 m+1} \text { for } m \geq 3 \\
\left\|f_{\mathbf{a}, \mathbf{0}, \mathbf{c}, \mathbf{h}, \mathbf{k}}^{\text {out }}\right\|_{C_{\delta}^{4, \alpha}\left(M_{\mathbf{p}, \mathbf{q}}\right) \oplus \mathcal{E}_{\mathbf{p}} \oplus \mathcal{E}_{\mathbf{q}} \oplus \mathcal{D}_{\mathbf{q}}(\overline{\mathbf{a}})} \leq \mathrm{Cr} r_{\varepsilon}^{5} \quad \text { for } m=2 .
\end{array}
$$

Moreover, $s_{\omega_{\mathbf{a}, 0, \mathbf{c}, \mathbf{h}, \mathbf{k}}}$ the scalar curvature of $\omega_{\mathbf{a}, \mathbf{0}, \mathbf{c}, \mathbf{h}, \mathbf{k}}$, is a small perturbation of $s_{\omega}$, the scalar curvature of the background metric $\omega$

$$
\left|s_{\omega_{\mathrm{a}, 0, \mathbf{c}, \mathbf{h}, \mathbf{k}}}-s_{\omega}\right| \leq \mathrm{C} \varepsilon^{2 m-2}
$$

The second one deals with the case $\mathbf{q}=\emptyset$. Proposition 4.4 follows from the same argument of the proof of [2, Proposition 5.4] observing that Ricci flatness does not enter in the proof.

Proposition 4.4. Let $(M, g, \omega)$ a Kcsc orbifold with isolated singularities, and let $\mathbf{p}$ be the set of singular points with nontrivial orbifold group that admit a scalar flat ALE resolution with $e(\Gamma)=0$.

- Assume exist $\mathbf{b} \in\left(\mathbb{R}^{+}\right)^{N}$ and $\mathbf{c} \in \mathbb{R}^{N}$ such that

$$
\begin{cases}\sum_{j=1}^{N} \frac{c\left(\Gamma_{j}\right)}{\left|c\left(\Gamma_{j}\right)\right|}\left(b_{j} \Delta_{\omega} \varphi_{i}\left(p_{j}\right)+c_{j} \varphi_{i}\left(p_{j}\right)\right)=0 & i=1, \ldots, d \\ (\Theta(\mathbf{b}, \mathbf{c}))_{\substack{1 \leq i \leq d \\ 1 \leq j \leq N}} & \text { has full rank, }\end{cases}
$$

where $(\Theta(\mathbf{b}, \mathbf{c}))_{\substack{1 \leq i \leq d \\ 1 \leq j \leq N}}$ is the matrix introduced in Section 3 formula (3.3). Let $\mathrm{G}_{0, \mathbf{b}, \mathbf{c}}$ be the multi-poles solution of $\mathbb{L}_{\omega}$ introduced in Section 3 in formula 3.3.

- Let $\delta \in(4-2 m, 5-2 m)$. Given any $(\mathbf{h}, \mathbf{k}) \in \mathcal{B}(\kappa, \delta)$, where $\mathcal{B}(\kappa, \delta)$ is the space defined in formula (4.2), let $\mathbf{H}_{\mathrm{h}, \mathrm{k}}^{\text {out }}$ be the function defined in formula (4.7).

$$
\mathbf{H}_{\mathbf{h}, \mathbf{k}}^{\text {out }}:=\sum_{j=1}^{N} \chi_{j} H_{h_{j}^{\text {out }}, k_{j}^{(\dagger)}}^{\left(\frac{z}{r_{\varepsilon}}\right) .}
$$


- Let $\mathbf{P}_{\mathbf{b}, \eta}$ be the transplanted potentials defined in formula (4.8)

$$
\mathbf{P}_{\mathbf{b}, \eta}:=\sum_{j=1}^{N} B_{j}^{2} \varepsilon^{2} \chi_{j} \psi_{\eta_{j}}\left(\frac{z}{B_{j} \varepsilon}\right) .
$$

Then there is $f_{\mathbf{0}, \mathbf{b}, \mathbf{c}, \mathbf{h}, \mathbf{k}}^{\text {out }} \in C_{\delta}^{4, \alpha}\left(M_{\mathbf{p}}\right) \oplus \mathcal{D}_{\mathbf{p}}(\mathbf{b}, \mathbf{c})$ if $m \geq 3$ and $f_{\mathbf{0}, \mathbf{b}, \mathbf{c}, \mathbf{h}, \mathbf{k}}^{\text {out }} \in C_{\delta}^{4, \alpha}\left(M_{\mathbf{p}}\right) \oplus \mathcal{E}_{\mathbf{p}} \oplus$ $\mathcal{D}_{\mathbf{p}}(\mathbf{b}, \mathbf{c})$ if $m=2$ such that

$$
\omega_{0, \mathbf{b}, \mathbf{c}, \mathbf{h}, \mathbf{k}}=\omega+i \partial \bar{\partial}\left(\mathbf{G}_{0, \mathbf{b}, \mathbf{c}}+\mathbf{P}_{\mathbf{b}, \eta}+\mathbf{H}_{\mathbf{h}, \mathbf{k}}^{\text {out }}+f_{0, \mathbf{b}, \mathbf{c}, \mathbf{h}, \mathbf{k}}^{\text {out }}\right)
$$

is a Kcsc metric on $M_{r_{\varepsilon}}$ and the following estimates hold.

$$
\begin{aligned}
\left\|f_{\mathbf{0}, \mathbf{b}, \mathbf{c}, \mathbf{h}, \mathbf{k}}^{\text {out }}\right\|_{C_{\delta}^{4, \alpha}\left(M_{\mathbf{p}}\right) \oplus \mathcal{D}_{\mathbf{p}}(\mathbf{b}, \mathbf{c})} & \leq \mathrm{C} \varepsilon^{2 m+2} r_{\varepsilon}^{2-2 m-\delta} \text { for } m \geq 3 \\
\left\|f_{\mathbf{0}, \mathbf{b}, \mathbf{c}, \mathbf{h}, \mathbf{k}}^{\text {out }}\right\|_{C_{\delta}^{4, \alpha}\left(M_{\mathbf{p}}\right) \oplus \mathcal{E}_{\mathbf{p}} \oplus \mathcal{D}_{\mathbf{p}}(\mathbf{b}, \mathbf{c})} & \leq \mathrm{C} \varepsilon^{6} r_{\varepsilon}^{-2-\delta} \text { for } m=2 .
\end{aligned}
$$

Moreover, $s_{\omega_{0, \mathbf{b}, \mathbf{c}, \mathbf{h}, \mathbf{k}}}$ the scalar curvature of $\omega_{0, \mathbf{b}, \mathbf{c}, \mathbf{h}, \mathbf{k},}$ is a small perturbation of $s_{\omega}$, the scalar curvature of the background metric $\omega$

$$
\left|s_{\omega_{0, \mathbf{b}, \mathbf{c}, \mathbf{h}, \mathbf{k}}}-s_{\omega}\right| \leq \mathrm{C} \varepsilon^{2 m}
$$

\subsection{Extremal metrics on the truncated model spaces}

As in the base case, we want to construct perturbations of $\eta$ on $X_{\Gamma, \frac{R_{\varepsilon}}{a}}$ of the form

$$
\eta_{\tilde{\mathbf{h}}, \tilde{\mathbf{k}}}:=\varepsilon^{2} a^{2} \eta+i \varepsilon^{2} \partial \bar{\partial} F_{\tilde{\mathbf{h}}, \tilde{\mathbf{k}}}^{\mathrm{inn}} \quad a \in \mathbb{R}^{+}
$$

such that $\tilde{\eta}_{\tilde{h}, \tilde{k}}$ is extremal. Again, by Proposition 2.3 , we need to find $\left(F_{\tilde{\mathbf{h}}, \tilde{\mathbf{k}}}^{\mathrm{inn}}, c^{\mathrm{inn}}\right) \in$ $C^{\infty}\left(X_{\Gamma, \frac{R_{\varepsilon}}{a}}\right)^{T} \times \mathbb{R}$ that solve equation (2.5), that is,

$$
\begin{aligned}
P_{\eta}^{*} P_{\eta}\left[F_{\tilde{\mathrm{h}}, \tilde{\mathrm{k}}}^{\mathrm{inn}}\right]= & -2 \varepsilon^{4} a^{4} c^{\mathrm{inn}}-2 \varepsilon^{4} a^{6}\left\langle\mu_{\eta}, \tilde{X}_{s}+\tilde{X}^{\text {out }}\right\rangle \\
& -\varepsilon^{4} a^{4} J\left(\tilde{X}_{s}+\tilde{X}^{\text {out }}\right)\left[F_{\tilde{\mathrm{h}}, \tilde{\mathrm{k}}}^{\mathrm{inn}}\right]+a^{2} \mathbb{N}_{\eta}\left(\frac{1}{a^{2}} F_{\tilde{\mathrm{h}}, \tilde{\mathrm{k}}}^{\text {inn }}\right)
\end{aligned}
$$

Remark 4.5. We want to point out that equation (2.4) on $X_{\Gamma, \frac{R_{e}}{a}}$ and consequently equation (4.9) make sense only because of Proposition 3.3. Indeed, to write equation (4.9), we need the moment map $\mu_{\eta}$ that produces Hamiltonian potentials of holomorphic vector fields on $X_{\Gamma}$ and Proposition 3.3 precisely ensures the existence of such Hamiltonian potentials. 
We look for $F_{\tilde{\mathbf{h}}, \tilde{\mathbf{k}}}^{\mathrm{inn}}$ of the form

$$
F_{\tilde{\mathbf{h}}, \tilde{\mathbf{k}}}^{\mathrm{inn}}:=\mathbf{H}_{\tilde{h}, \tilde{k}}^{\mathrm{inn}}+f_{\tilde{\mathbf{h}}, \tilde{\mathbf{k}}}^{\mathrm{inn}}
$$

with

$$
\mathbf{H}_{\tilde{h}, \tilde{k}}^{\mathrm{inn}}:=\chi H_{\tilde{h}, \tilde{k}}^{\operatorname{inn}}\left(\frac{a x}{R_{\varepsilon}}\right)
$$

As usual, it is convenient to work on the complete model $X_{\Gamma}$ and to use the standard truncation/extension operators $\mathcal{E}_{R_{\varepsilon}}$, so we want to find $\left(f_{\tilde{\mathbf{h}}, \tilde{\mathbf{k}}}^{\text {inn }}, c^{\text {inn }}\right) \in C_{\delta}^{4, \alpha}\left(X_{\Gamma}\right)^{T} \times \mathbb{R}$ with $\delta \in(0,1)$ such that

$$
\begin{aligned}
P_{\eta}^{*} P_{\eta}\left[f_{\tilde{\mathbf{h}}, \tilde{\mathrm{k}}}^{\mathrm{inn}}\right]= & -\mathcal{E}_{\frac{R_{\varepsilon}}{a}} P_{\eta}^{*} P_{\eta}\left[\mathbf{H}_{\tilde{h}, \tilde{k}}^{\mathrm{inn}}\right]-2 \mathcal{E}_{\frac{R_{\varepsilon}}{a}} \varepsilon^{4} a^{4} c^{\mathrm{inn}}-2 \varepsilon^{4} a^{6} \mathcal{E}_{\frac{R_{\varepsilon}}{a}}\left\langle\mu_{\eta}, X\right\rangle \\
& -\varepsilon^{4} a^{4} \mathcal{E}_{\frac{R_{\varepsilon}}{a}} J\left(\tilde{X}_{S}+\tilde{X}^{\text {out }}\right)\left[\mathbf{H}_{\tilde{h}, \tilde{k}}^{\mathrm{inn}}+f_{\tilde{\mathbf{h}}, \tilde{\mathrm{k}}}^{\mathrm{inn}}\right]+a^{2} \mathcal{E}_{\frac{R_{\varepsilon}}{a}} \mathbb{N}_{\eta}\left(\frac{1}{a^{2}}\left(\mathbf{H}_{\tilde{h}, \tilde{k}}^{\mathrm{inn}}+f_{\tilde{\mathbf{h}}, \tilde{\mathbf{k}}}^{\mathrm{inn}}\right)\right) .
\end{aligned}
$$

Using Proposition 3.2, we can we rephrase equation (4.10) as a fixed point problem

$$
\left(f_{\tilde{\mathbf{h}}, \tilde{\mathbf{k}}}^{\mathrm{inn}}, c^{\mathrm{inn}}\right)=\mathcal{N}^{\mathrm{inn}}\left(f_{\tilde{\mathbf{h}}, \tilde{\mathbf{k}}}^{\mathrm{inn}}, c^{\mathrm{inn}}, \tilde{\mathbf{h}}, \tilde{\mathbf{k}}\right)
$$

with

$$
\mathcal{N}^{\text {inn }}: C_{\delta}^{4, \alpha}\left(X_{\Gamma}\right)^{T} \times \mathbb{R} \times \mathcal{B}(\kappa, \delta) \rightarrow C_{\delta}^{4, \alpha}\left(X_{\Gamma}\right)^{T} \times \mathbb{R}
$$

nonlinear continuous operator. With computations analogous to those performed in [4], it is possible to obtain the following result.

Proposition 4.6. Let $\left(X_{\Gamma}, h, \eta\right)$ be a scalar-flat ALE resolutions of $\mathbb{C}^{m} / \Gamma$ with $T$-invariant metric $h$. Then for every $\left(\varepsilon^{2} \tilde{h}, \varepsilon^{2} \tilde{k}\right) \in \mathcal{B}(\kappa, \delta)$ and $a \in \mathbb{R}^{+}$, there is $\left(f_{\tilde{\mathbf{h}}, \tilde{\mathbf{k}}}^{\mathrm{inn}}, c^{\mathrm{inn}}\right) \in C_{\delta}^{4, \alpha}\left(X_{\Gamma}\right)^{T} \times \mathbb{R}$ with

$$
\left\|f_{\tilde{\mathbf{h}}, \tilde{\mathbf{k}}}^{\mathrm{inn}}\right\|_{C_{\delta}^{4, \alpha}\left(X_{\Gamma}\right)}+\left|C^{\mathrm{inn}}\right| \leq C(\kappa) R_{\varepsilon}^{4-2 m}
$$

such that

$$
\eta_{\mathbf{h}, \mathbf{k}}:=a^{2} \varepsilon^{2} \eta+i \varepsilon^{2} \partial \bar{\partial}\left(\mathbf{H}_{\tilde{h}, \tilde{k}}^{\mathrm{inn}}+f_{\tilde{\mathbf{h}}, \tilde{\mathbf{k}}}^{\mathrm{inn}}\right)
$$

is an extremal Kähler metric on $X_{\Gamma, \frac{R_{e}}{a}}$ with extremal vector field $\tilde{X}_{s}+\tilde{X}^{\text {out }}$ which is the natural lift of the vector field $X_{S}+X^{\text {out }}$ defined in Proposition 4.2. 
As for the base case, an important consequence of Proposition 4.6 is that the following estimate holds

$$
\|\left.\left.\varepsilon^{2} f_{\tilde{\mathbf{h}}, \tilde{\mathbf{k}}}^{\mathrm{inn}}\left(\frac{R_{\varepsilon}}{a} \cdot\right)\right|_{X_{\Gamma} \backslash X_{\Gamma, \frac{R_{\varepsilon}}{2 a}}}\right|_{C^{4, \alpha}\left(\bar{B}_{1} \backslash B_{\frac{1}{2}}\right)} \leq \mathrm{C} r_{\varepsilon}^{4}
$$

with C positive constant depending only on $g, \eta$. Again, this kind of estimate is necessary for the success of the data-matching procedure.

\subsection{Kcsc metrics on the truncated model spaces}

We now want to perform, on the model spaces $X_{\Gamma_{j}}$ 's and $Y_{\Gamma_{N+l}}$, a similar analysis as in the previous subsection. The constructions of the families of metrics are essentially the same made in [2] except for some complications due to the fact we require the models to be only scalar flat with $e(\Gamma)=0$ and not necessarily Ricci flat. These technical complications show up when we construct the transplanted potential and the extensions of pseudo boundary data and are due to the presence of coefficients $c_{0}, c_{2}, c_{3}, c_{4}, c_{5}$ relative to particular asymptotics of the potentials at infinity of the families of metrics which in the Ricci-flat case could be taken $c_{0}=C_{2}=C_{3}=C_{5}=0$, and $C_{4}=-\frac{4(m-1)^{2} S_{\omega}}{m(m+1)}$ . These coefficients, as we will see in the last section will influence the balancing condition.

Notation. To keep notations as short as possible, we drop the subscripts $j$ and $l$.

Our starting point are scalar-flat ALE Kähler manifold $\left(X_{\Gamma}, \eta, h\right)$ and $\left(Y_{\Gamma}, \theta, k\right)$, where we want to find $F_{\tilde{b}, \tilde{h}, \tilde{k}}^{\text {in }} \in C^{4, \alpha}\left(X_{\Gamma, \frac{R_{\varepsilon}}{b}}\right)$, respectively, $F_{\tilde{a}, \tilde{h}, \tilde{k}}^{\text {in }} \in C^{4, \alpha}\left(Y_{\Gamma, \frac{R_{\varepsilon}}{a}}\right)$ with $\tilde{a}, \tilde{b} \in \mathbb{R}^{+}$ such that

$$
\theta_{\tilde{a}, \tilde{h}, \tilde{k}}:=\tilde{a}^{2} \theta+\mathrm{i} \partial \bar{\partial} F_{\tilde{a}, \tilde{h}, \tilde{k}}^{\text {in }}
$$

and

$$
\eta_{\tilde{b}, \tilde{h}, \tilde{k}}:=\tilde{b}^{2} \eta+\mathrm{i} \partial \bar{\partial} F_{\tilde{b}, \tilde{h}, \tilde{k}}^{\text {in }}
$$

are metrics on $Y_{\Gamma, \frac{R_{\varepsilon}}{\bar{a}}}$ and $X_{\Gamma, \frac{R_{\varepsilon}}{\bar{b}}}$. Moreover,

$$
\mathbf{S}_{\tilde{a}^{2} \theta}\left(F_{\tilde{a}, \tilde{h}, \tilde{k}}^{\text {in }}\right)=\mathbf{S}_{\eta}\left(F_{\tilde{b}, \tilde{h}, \tilde{k}}^{\text {in }}\right)=\varepsilon^{2}\left(s_{\omega}+\frac{1}{2} S_{\mathbf{a}, \mathbf{0}, \mathbf{c}, \mathbf{h}, \mathbf{k}}\right)
$$

when $\mathbf{q} \neq \varnothing$ and

$$
\mathbf{S}_{\tilde{b}^{2} \eta}\left(F_{\tilde{a}, \tilde{h}, \tilde{k}}^{\text {in }}\right)=\mathbf{S}_{\tilde{b}^{2} \eta}\left(F_{\tilde{b}, \tilde{h}, \tilde{k}}^{\text {in }}\right)=\varepsilon^{2}\left(s_{\omega}+\frac{1}{2} S_{0, \mathbf{b}, \mathbf{c}, \mathbf{h}, \mathbf{k}}\right)
$$


when $\mathbf{q}=\emptyset$ with $\mathbf{S}$. the operator introduced in (2.6).

The parameters $\tilde{a}, \tilde{b}$ with the "manual tuning" of the principal asymptotics and $\tilde{h}, \tilde{k}$ with the Cauchy data-matching procedure. The modifications of the metrics $\eta$ and $\theta$ will be made of blocks and it will take different shapes if $\mathbf{q} \neq \emptyset$ or if $\mathbf{q}=\emptyset$. Indeed, if $\mathbf{q} \neq \emptyset$ then on $Y_{\Gamma}$

$$
F_{\tilde{a}, \tilde{h}, \tilde{k}}^{\text {in }}:=\mathbf{H}_{\tilde{h}, \tilde{k}}^{\text {in }}+f_{\tilde{a}, \tilde{h}, \tilde{k}}^{\text {in }}
$$

and on $X_{\Gamma}$

$$
F_{\tilde{b}, \tilde{h}, \tilde{k}}^{\text {in }}:=\mathbf{H}_{\tilde{h}, \tilde{k}}^{\text {in }}+f_{\tilde{b}, \tilde{h}, \tilde{k}}^{\text {in }},
$$

if instead $\mathbf{q}=\emptyset$, then

$$
F_{\tilde{b}, \tilde{h}, \tilde{k}}^{\text {in }}:=\mathbf{P}_{\tilde{b}, \omega}+\mathbf{H}_{\tilde{h}, \tilde{k}}^{\text {in }}+f_{\tilde{b}, \tilde{h}, \tilde{k}}^{\text {in }}
$$

The term $\mathbf{P}_{\tilde{b}, \omega}$ is the transplanted potential of $\omega$ and comes into play when there are only $X_{\Gamma}$ 's, $\mathbf{H}_{\tilde{h}, \tilde{k}}^{\text {in }}$ is the biharmonic extension of pseudo-boundary data $f_{\tilde{a}, \tilde{h}, \tilde{k}}^{\text {in }}, f_{\tilde{b}, \tilde{h}, \tilde{k}}^{\text {in }}$ are the perturbations ensuring the constancy of the scalar curvature.

Transplanted potential. If $\mathbf{q}=\emptyset$, then we introduce the term $\mathbf{P}_{\tilde{b}, \omega}$ that is a suitable modification of the function $\psi_{\omega}$. Following exactly the same strategy of [2], we look for functions $W_{4}, W_{5}$ solutions of

$$
\begin{aligned}
& \mathbb{L}_{\eta}\left[\Psi_{4}+W_{4}\right]=-2 s_{\omega} \\
& \mathbb{L}_{\eta}\left[\Psi_{5}+W_{5}\right]=0 .
\end{aligned}
$$

Notation. For the rest of the subsection, $\chi$ will denote a smooth cutoff function identically 0 on $X_{\Gamma, \frac{R_{0}}{3 \bar{b}}}$ and identically 1 outside $X_{\Gamma, \frac{R_{0}}{2 \bar{b}}}$.

We set

$$
\begin{aligned}
& u_{4}:= \begin{cases}\left(\frac{\Phi_{2}}{\Lambda_{2}^{2}}+\frac{\Phi_{4}}{\Lambda_{4}^{2}}\right) \chi|x|^{4-2 m} & \text { for } m \geq 3 \\
\left(\frac{\Phi_{2}}{\Lambda_{2}^{2}}+\frac{\Phi_{4}}{\Lambda_{4}^{2}}\right) \chi \log (|x|) & \text { for } m=2\end{cases} \\
& u_{5}:=\left(\frac{\Phi_{3}}{\Lambda_{3}^{3}}+\frac{\Phi_{5}}{\Lambda_{5}^{2}}\right) \chi|x|^{5-2 m}
\end{aligned}
$$


for a suitable choice of $\Phi_{2}, \Phi_{4}, \Phi_{3}, \Phi_{5}$ eigenfunctions relative to the eigenvalues $\Lambda_{2}, \Lambda_{4}, \Lambda_{3}, \Lambda_{5}$ of $\Delta_{\mathbb{S} 2 m-1}$. Setting also

$$
\begin{aligned}
& \mathrm{C}_{4}:=\frac{|\Gamma|}{C(\Gamma)\left|\mathbb{S}^{2 m-1}\right|} \int_{X_{\Gamma}}\left(\mathbb{L}_{\eta}\left[\chi \Psi_{4}+u_{4}\right]+2 s_{\omega}\right) \mathrm{d} \mu_{\eta} \\
& \mathrm{C}_{5}:=\frac{|\Gamma|}{C(\Gamma)\left|\mathbb{S}^{2 m-1}\right|} \int_{X_{\Gamma}} \mathbb{L}_{\eta}\left[\chi \Psi_{5}+u_{5}\right] \mathrm{d} \mu_{\eta}
\end{aligned}
$$

we can find $v_{4} \in C_{\delta}^{4, \alpha}\left(X_{\Gamma}\right)$ with $\delta \in(2-2 m, 3-2 m)$ and $v_{5} \in C_{\delta}^{4, \alpha}\left(X_{\Gamma}\right)$ with $\delta \in$ $(3-2 m, 4-2 m)$ such that

$$
\begin{aligned}
\mathbb{L}_{\eta}\left[\chi \Psi_{4}+u_{4}-\frac{c(\Gamma) c_{4}}{8(m-2)(m-1)} \chi|X|^{4-2 m}+v_{4}\right]=-2 s_{\omega} & \text { for } m \geq 3 \\
\mathbb{L}_{\eta}\left[\chi \Psi_{4}+u_{4}+\frac{c(\Gamma) c_{4}}{4} \chi \log (|x|)+v_{4}\right]=-2 s_{\omega} & \text { for } m=2
\end{aligned}
$$

Remark 4.7. Contrary to [2], here we do not have any information on constants $\mathrm{C}_{4}, \mathrm{C}_{5}$, we do not know even their sign. If $X_{\Gamma}$ is Ricci flat, as in [2], one can to compute explicitly the constants $c_{4}, c_{5}$ and show that $c_{5}=0$ and $c_{4}$ depends linearly on the scalar curvature of $M$ and nonlinearly on the dimension.

Now we can write the explicit expression of $W_{4}$

$$
W_{4}:= \begin{cases}-\frac{c(\Gamma) c_{4} \tilde{b}^{4} \varepsilon^{2}}{8(m-2)(m-1)} \chi|x|^{4-2 m}+u_{4}+v_{4} & \text { for } m \geq 3, \\ \frac{c(\Gamma) c_{4} \tilde{b}^{4} \varepsilon^{2}}{4} \chi \log (|x|)+u_{4}+v_{4} & \text { for } m=2 .\end{cases}
$$

Analogous to the case of $\Psi_{4}$, the correction $W_{5}$ of $\Psi_{5}$ is then

$$
W_{5}:= \begin{cases}-\frac{c(\Gamma) c_{5} \tilde{b}^{5} \varepsilon^{3}}{8(m-2)(m-1)} \chi|x|^{4-2 m}+u_{5}+v_{5} & \text { for } m \geq 3, \\ \frac{c(\Gamma) c_{5} \tilde{b}^{5} \varepsilon^{3}}{4} \chi \log (|x|)+u_{5}+v_{5} & \text { for } m=2 .\end{cases}
$$

If we define

$$
V:=\varepsilon^{2} \tilde{b}^{4} W_{4}+\varepsilon^{3} \tilde{b}^{5} W_{5}
$$


then we can define the transplanted potential $\mathbf{P}_{\tilde{b}, \omega}$ as the function in $C^{4, \alpha}\left(X_{\Gamma, \frac{R_{\varepsilon}}{b}}\right)$

$$
\mathbf{P}_{\tilde{b}, \omega}:= \begin{cases}\frac{1}{\varepsilon^{2}} \chi \psi_{\omega}(\tilde{b} \varepsilon x)+V & \text { for } m \geq 3, \\ \frac{1}{\varepsilon^{2}} \chi \psi_{\omega}(\tilde{b} \varepsilon x)+V+C & \text { for } m=2,\end{cases}
$$

where $C$ is the constant term in the expansion at $B_{2 r_{0}}(p) \backslash B_{r_{\varepsilon}}(p)$ of

$$
F_{\mathbf{0}, \mathbf{b}, \mathbf{c}, \mathbf{h}, \mathbf{k}}^{\text {out }}=-\varepsilon^{2 m} \mathbf{G}_{\mathbf{0 , \mathbf { b } , \mathbf { c }}}+\mathbf{P}_{\mathbf{b}, \boldsymbol{\eta}}+\mathbf{H}_{\mathbf{h}, \mathbf{k}}^{\text {out }}+f_{0, \mathbf{b}, \mathbf{c}, \mathbf{h}, \mathbf{k}}^{\text {out }} .
$$

introduced in Proposition 4.4.

Extensions of pseudo-boundary data. Also this term takes different forms whether $\mathbf{q}=\emptyset$ or not. If $\mathbf{q} \neq \emptyset$, then we define $\mathbf{H}_{\tilde{h}, \tilde{k}}^{\text {in }} \in C^{4, \alpha}\left(Y_{\Gamma}, \frac{R_{\varepsilon}}{\tilde{a}}\right)$ and $\mathbf{H}_{\tilde{h}, \tilde{k}}^{\text {in }} \in$ $C^{4, \alpha}\left(X_{\Gamma}, R_{\varepsilon}\right)$ as

$$
\mathbf{H}_{\tilde{h}, \tilde{k}}^{\text {in }}:=H_{\tilde{h}, \tilde{k}}^{\text {in }}(0)+\chi\left(H_{\tilde{h}, \tilde{k}}^{\text {in }}\left(\frac{\tilde{b} X}{R_{\varepsilon}}\right)-H_{\tilde{h}, \tilde{k}}^{\text {in }}(0)\right) .
$$

If instead $\mathbf{q}=\emptyset$, we need the construction of $\mathbf{H}_{\tilde{h}, \tilde{k}}^{\mathrm{in}}$ performed in [2]. Indeed, as for the transplanted potential, we look for functions $W_{0}, W_{2}, W_{3}$ for the equations

$$
\begin{aligned}
\mathbb{L}_{\eta}\left[\chi|X|^{2}+W_{0}\right] & =0, \\
\mathbb{L}_{\eta}\left[\chi|X|^{2} \Phi_{2}+W_{2}\right] & =0, \\
\mathbb{L}_{\eta}\left[\chi|X|^{3} \Phi_{3}+W_{3}\right] & =0 .
\end{aligned}
$$

with $W_{0}, W_{2}, W_{3}$ having a structure similar to $W_{4}, W_{5}$ we built for the transplanted potential. Indeed, once we set

$$
\begin{aligned}
& \mathrm{C}_{0}:=\frac{|\Gamma|}{C(\Gamma)\left|\mathbb{S}^{2 m-1}\right|} \int_{X_{\Gamma}} \mathbb{L}_{\eta}\left[\chi|x|^{2}\right] d \mu_{\eta} \\
& \mathrm{C}_{2}:=\frac{|\Gamma|}{C(\Gamma)\left|\mathbb{S}^{2 m-1}\right|} \int_{X_{\Gamma}} \mathbb{L}_{\eta}\left[\chi|x|^{2} \Phi_{2}\right] d \mu_{\eta} \\
& \mathrm{C}_{3}:=\frac{|\Gamma|}{C(\Gamma)\left|\mathbb{S}^{2 m-1}\right|} \int_{X_{\Gamma}} \mathbb{L}_{\eta}\left[\chi|x|^{3} \Phi_{3}+u^{(3)}\right] d \mu_{\eta}
\end{aligned}
$$

with

$$
u^{(3)}:=\chi|x|^{3-2 m} \tilde{\Phi}_{3}
$$


for a suitable spherical harmonic $\tilde{\Phi}_{3}$, we define

$$
\begin{aligned}
& W_{0}:=\left\{\begin{array}{lc}
-\frac{c(\Gamma) c_{0}}{8(m-2)(m-1)} \chi|X|^{4-2 m}+v^{(0)} & m \geq 3 \\
\frac{c(\Gamma) c_{0}}{4} \chi|X|^{4-2 m}+v^{(0)} & m=2
\end{array}\right. \\
& W_{2}:= \begin{cases}\frac{c(\Gamma) c_{2}}{8(m-2)(m-1)}|X|^{4-2 m}+v^{(2)} & m \geq 3 \\
-\frac{c(\Gamma) c_{2}}{8} \chi|X|^{4-2 m}+v^{(2)} & m=2\end{cases} \\
& W_{3}:= \begin{cases}-\frac{c(\Gamma) c_{3}}{8(m-2)(m-1)}|x|^{4-2 m}+u^{(3)}+v^{(3)} & m \geq 3 \\
\frac{c(\Gamma) c_{3}}{4}|x|^{4-2 m}+u^{(3)}+v^{(3)} & m \geq 3\end{cases}
\end{aligned}
$$

with $v^{(0)}, v^{(2)}, v^{(3)} \in C_{\delta}^{4, \alpha}\left(X_{\Gamma}\right)$ for $\delta \in(2-2 m, 3-2 m)$.

Remark 4.8. Here we see a technical complication due to the fact we ask $X_{\Gamma}$ to be only scalar flat with $e(\Gamma)=0$ and not Ricci flat. Indeed, if $X_{\Gamma}$ were Ricci flat, as in [2], then the constants $\mathrm{C}_{0}, \mathrm{C}_{2}, \mathrm{C}_{3}$ would vanish, making the behavior of the $W_{j}$ 's easier.

Moreover, we set

$$
V^{\prime}:=\frac{\tilde{k}^{(0)} \tilde{b}^{2}}{4 m R_{\varepsilon}^{2}} W_{0}+\left(\tilde{h}^{(2)}-\frac{\tilde{k}^{(2)}}{4(m+2)}\right) \frac{\tilde{b}^{2}}{R_{\varepsilon}^{2}} W_{2}+\frac{\tilde{b}^{3}}{R_{\varepsilon}^{3}}\left(\tilde{h}^{(3)}-\frac{\tilde{k}^{(3)}}{4(m+3)}\right) W_{3}
$$

and hence we can introduce the function $\mathbf{H}_{\tilde{h}, \tilde{k}}^{\text {in }} \in C^{4, \alpha}\left(X_{\Gamma, \frac{R_{\varepsilon}}{b}}\right)$

$$
\mathbf{H}_{\tilde{h}, \tilde{k}}^{\text {in }}:=H_{\tilde{h}, \tilde{k}}^{\text {in }}(0)+\chi\left(H_{\tilde{h}, \tilde{k}}^{\text {in }}\left(\frac{\tilde{b} x}{R_{\varepsilon}}\right)-H_{\tilde{h}, \tilde{k}}^{\text {in }}(0)\right)+V^{\prime} .
$$

Correction term. The terms $f_{\tilde{a}, \tilde{h}, \tilde{k}}^{\text {in }}$ and $f_{\tilde{b}, \tilde{h}, \tilde{k}}^{\text {in }}$ that ensure the constancy of the scalar curvature of the metrics $\theta_{\tilde{a}, \tilde{h}, \tilde{k}}$ and of $\eta_{\tilde{b}, \tilde{h}, \tilde{k}}$ on $X_{\Gamma, R_{\varepsilon}}$ and are solutions of a fixed point problem on a suitable closed and bounded subspace of $C_{\delta}^{4, \alpha}\left(X_{\Gamma}\right)$.

We are now ready to state the main results on the model spaces.

Proposition 4.9. Let $\mathbf{q} \neq \emptyset$ and let $\left(X_{\Gamma}, h, \eta\right)$ and $\left(Y_{\Gamma}, k, \theta\right)$ ALE scalar-flat Kähler resolutions of $\mathbb{C}^{m} / \Gamma$ with $\Gamma$ finite subgroup of $U(m)$. Let $\delta \in(0,1)$. Given any $(\tilde{h}, \tilde{k}) \in \mathcal{B}$, such that $\left(\varepsilon^{2} \tilde{h}, \varepsilon^{2} \tilde{k}\right) \in \mathcal{B}(\kappa, \delta)$, where $\mathcal{B}(\kappa, \delta)$ is the space defined in formula (4.2), let $\mathbf{H}_{\tilde{h}, \tilde{k}}^{\text {in }}$ be the 
function defined in formula (4.13).

$$
\mathbf{H}_{\tilde{h}, \tilde{k}}^{\text {in }}:=H_{\tilde{h}, \tilde{k}}^{\text {in }}(0)+\chi\left(H_{\tilde{h}, \tilde{k}}^{I}\left(\frac{\tilde{b} X}{R_{\varepsilon}}\right)-H_{\tilde{h}, \tilde{k}}^{I}(0)\right) .
$$

Then there is $f_{\tilde{a}, \tilde{h}, \tilde{k}}^{\mathrm{in}} \in C_{\delta}^{4, \alpha}\left(Y_{\Gamma}\right)$ and $f_{\tilde{b}, \tilde{h}, \tilde{k}}^{\mathrm{in}} \in C_{\delta}^{4, \alpha}\left(X_{\Gamma}\right)$ such that

$$
\theta_{\tilde{a}, \tilde{h}, \tilde{k}}=\tilde{a}^{2} \theta+\mathrm{i} \partial \bar{\partial}\left(\mathbf{H}_{\tilde{h}, \tilde{k}}^{\text {in }}+f_{\tilde{a}, \tilde{h}, \tilde{k}}^{\text {in }}\right)
$$

is a $\operatorname{Kcsc}$ metric on $Y_{\Gamma, \frac{R_{\varepsilon}}{\bar{a}}}$ and

$$
\eta_{\tilde{h}, \tilde{k}}=\tilde{b}^{2} \eta+i \partial \bar{\partial}\left(\mathbf{H}_{\tilde{h}, \tilde{k}}^{\text {in }}+f_{\tilde{b}, \tilde{h}, \tilde{k}}^{\text {in }}\right)
$$

is a Kcsc metric on $X_{\Gamma, \frac{R_{\varepsilon}}{b}}$. Moreover, the following estimates hold.

$$
\left\|f_{\tilde{b}, \tilde{h}, \tilde{k}}^{\mathrm{in}}\right\|_{C_{\delta}^{4, \alpha}\left(X_{\Gamma}\right)},\left\|f_{\tilde{a}, \tilde{h}, \tilde{k}}^{\mathrm{in}}\right\|_{C_{\delta}^{4, \alpha}\left(Y_{\Gamma}\right)} \leq \mathrm{C} \varepsilon^{2} R_{\varepsilon}^{4-\delta}
$$

Moreover, $s_{\eta_{\tilde{b}, \tilde{h}, \tilde{k}}}$ and $s_{\theta_{\tilde{a}, \tilde{h}, \tilde{k}}}$ satisfy

$$
s_{\eta_{\tilde{h}, \tilde{k}}}=s_{\theta_{\tilde{a}, \tilde{h}, \tilde{k}}}=s_{\omega_{\mathbf{a}, 0, \mathbf{c}, \mathbf{h}, \mathbf{k}}} .
$$

The proof of Proposition 4.9 is an easy adaptation of [3, Lemma 5.3 ].

Proposition 4.10. Let $\mathrm{q}=\emptyset$ and let $\left(X_{\Gamma}, h, \eta\right)$ be a scalar flat ALE Kähler resolution of $\mathbb{C}^{m} / \Gamma$ with $\Gamma$ finite subgroup of $U(m)$ and $e(\Gamma)=0$.

- Let $\delta \in(4-2 m, 5-2 m)$. Given any $(\tilde{h}, \tilde{k}) \in \mathcal{B}$, such that $\left(\varepsilon^{2} \tilde{h}, \varepsilon^{2} \tilde{k}\right) \in \mathcal{B}(\kappa, \delta)$, where $\mathcal{B}(\kappa, \delta)$ is the space defined in formula (4.2), let $\mathbf{H}_{\tilde{h}, \tilde{k}}^{\text {in }}$ be the function defined in formula (4.14).

$$
\mathbf{H}_{\tilde{h}, \tilde{k}}^{\mathrm{in}}:=H_{\tilde{h}, \tilde{k}}^{\mathrm{in}}(0)+\chi\left(H_{\tilde{h}, \tilde{k}}^{\text {in }}\left(\frac{\tilde{b} x}{R_{\varepsilon}}\right)-H_{\tilde{h}, \tilde{k}}^{\text {in }}(0)\right)+V^{\prime} .
$$

- Let $\mathbf{P}_{\tilde{b}, \omega}$ be the transplanted potential defined in formula (4.12)

$$
\mathbf{P}_{\tilde{b}, \omega}:= \begin{cases}\frac{1}{\varepsilon^{2}} \chi \psi_{\omega}(\tilde{b} \varepsilon x)+V & \text { for } m \geq 3, \\ \frac{1}{\varepsilon^{2}} \chi \psi_{\omega}(\tilde{b} \varepsilon x)+V+C & \text { for } m=2 .\end{cases}
$$


Then there is $f_{\tilde{b}, \tilde{h}, \tilde{k}}^{\text {in }} \in C_{\delta}^{4, \alpha}\left(X_{\Gamma}\right)$ such that

$$
\eta_{\tilde{b}, \tilde{h}, \tilde{k}}=\tilde{b}^{2} \eta+i \partial \bar{\partial}\left(\mathbf{P}_{\tilde{b}, \omega}+\mathbf{H}_{\tilde{h}, \tilde{k}}^{\text {in }}+f_{\tilde{b}, \tilde{h}, \tilde{k}}^{\text {in }}\right)
$$

is a Kcsc metric on $X_{\Gamma, \frac{R_{e}}{b}}$ and the following estimate holds.

$$
\left\|f_{\tilde{b}, \tilde{h}, \tilde{k}}^{\text {in }}\right\|_{C_{\delta}^{4, \alpha}\left(X_{\Gamma}\right)} \leq C(\kappa) \varepsilon^{2 m+4} r_{\varepsilon}^{-4 m-\delta} R_{\varepsilon}^{-2}
$$

with $C(\kappa) \in \mathbb{R}^{+}$depending only on $\omega$ and $\eta_{j}$ 's and $\kappa$ the constant appearing in the definition of $\mathcal{B}(\kappa, \delta)$ (Section 4.1 formula 4.2 ). Moreover, $s_{\eta_{\tilde{b}, \tilde{b} . \bar{k}^{\prime}}}$ the scalar curvature of $\eta_{\tilde{b}, \tilde{h}, \tilde{k}}$ is

$$
s_{\eta_{\tilde{b}, \tilde{h}, \tilde{k}}}=s_{\omega_{0, \mathrm{~b}, \mathrm{~h}, \mathrm{k}}} \cdot
$$

The proof of Proposition 4.10 follows observing that the estimates in Lemmata 5.13-5.15 in [2] also hold in this case precisely due to the choice of $c_{0}, c_{2}, c_{3}, c_{5}$.

\section{Data Matching}

\subsection{The extremal case}

We can now complete the proof of the following.

Theorem 5.1. Let $(M, g, \omega)$ be a compact extremal orbifold with $T$-invariant metric $g$. Let $\mathbf{p}, \mathbf{q}$ be as above. Then there is $\bar{\varepsilon}$ such that for every $\varepsilon \in(0, \bar{\varepsilon})$ and $\mathbf{b} \in\left(\mathbb{R}^{+}\right)^{N}$ and $\mathbf{a} \in$ $\left(\mathbb{R}^{+}\right)^{K}$ the orbifold

$$
\tilde{M}:=M \sqcup_{p_{1}, \varepsilon} X_{\Gamma_{1}} \sqcup_{p_{2}, \varepsilon} \cdots \sqcup_{p_{N}, \varepsilon} X_{\Gamma_{N}} \sqcup_{q_{1}, \varepsilon} Y_{\Gamma_{N+1}} \sqcup_{q_{2}, \varepsilon} \cdots \sqcup_{q_{K}, \varepsilon} Y_{\Gamma_{N+K}}
$$

has a $\tilde{T}$-invariant extremal Kähler metric in the class

$$
\pi^{*}[\omega]+\sum_{l=1}^{K} \varepsilon^{2 m-2} \tilde{a}_{l}^{2 m-2}\left[\tilde{\theta}_{l}\right]+\sum_{j=1}^{N} \varepsilon^{2 m} b_{j}\left[\tilde{\eta}_{j}\right],
$$

where $\mathfrak{i}_{l}^{*}\left[\tilde{\theta}_{l}\right]=\left[\theta_{l}\right]$ with $\mathfrak{i}_{l}: Y_{\Gamma_{N+l}, R_{\varepsilon}} \hookrightarrow \tilde{M}$ the standard inclusion (and analogously for $\tilde{\eta}_{j}$ ).

Proof of Theorem 5.1. The result follows combining Proposition 4.2, Proposition 4.6, and the standard procedure of data matching exposed in [4, Section 10]. 


\subsection{The Kcsc case}

The final step is now to show a matching condition for the metrics constructed above, and in particular why the quantities $c_{0, j}, c_{2, j}, c_{3, j}, c_{5, j}$ do not interfere with the fixed point argument.

Proof of Theorem 1.2. We focus on the case $m \geq 3$ since the proof for the case $m=2$ is exactly the same. We denote with $\mathcal{V}_{j, \mathbf{a}, \mathbf{0}, \mathbf{c}, \mathbf{h}, \mathbf{k}}^{\text {out }}$ and $\mathcal{V}_{l, \mathbf{a}, \mathbf{0}, \mathbf{c}, \mathbf{h}, \mathbf{k}}^{\text {ou }}$ the Kähler potentials of $\omega_{\mathbf{a}, \mathbf{0}, \mathbf{c}, \mathbf{h}, \mathbf{k}}$ on neighborhoods of points $p_{j}$ and $q_{l}$, with $\mathcal{V}_{l, \tilde{a}_{l}, \tilde{h}_{l}, \tilde{\kappa}_{l}}^{\text {in }}$ the Kähler potential of $\theta_{\tilde{a}_{l}, \tilde{h}_{l}, \tilde{k}_{l}}$ on $Y_{\Gamma_{N+l}, \frac{R_{e}}{a_{l}}}$ and with $\mathcal{V}_{j, \tilde{b}_{j}, \tilde{h}_{j}, \tilde{k}_{l}}^{\text {in }}$ the Kähler potential of $\eta_{\tilde{b}_{j}, \tilde{h}_{j}, \tilde{k}_{j}}$ on $X_{\Gamma_{j}, \frac{R_{e}}{a_{j}}}$. It is possible, using Propositions 4.3 and 4.9 , to rescale, expand, and decompose the potentials $\mathcal{V}_{j, \mathbf{a}, \mathbf{0}, \mathbf{c}, \mathbf{h}, \mathbf{k}^{\prime}}^{\text {out }}$ $\mathcal{V}_{l, \mathbf{a}, \mathbf{0}, \mathbf{c}, \mathbf{h}, \mathbf{k}}^{\text {out }}, \mathcal{V}_{l, \tilde{a}_{l}, \tilde{h}_{l}, \tilde{k}_{l}}^{\text {in }} \mathcal{V}_{j, \tilde{b}_{j}, \tilde{h}_{j}, \tilde{k}_{l}}^{\text {in }}$ in the same way as done in [2].

We start with the case $\mathbf{q} \neq \varnothing$ and the "tuning conditions" are

$$
\begin{aligned}
e\left(\Gamma_{l}\right) \tilde{a}_{l}^{2 m-2} \varepsilon^{2} R_{\varepsilon}^{4-2 m} & =\left(1+\frac{\left(f_{\mathbf{a}, \mathbf{0}, \mathbf{c}, \mathbf{h}, \mathbf{k}}^{\text {out }}\right)^{l}}{\varepsilon^{2 m-2}}\right) e\left(\Gamma_{l}\right) \bar{a}_{l} \varepsilon^{2 m-2} r_{\varepsilon}^{4-2 m}-\frac{k_{l}^{(0)}}{4 m-8} \\
C_{j} \varepsilon^{2 m-2} r_{\varepsilon}^{4-2 m} & =\frac{k_{j}^{(0)}}{4 m-8},
\end{aligned}
$$

where the quantities $\left(f_{\mathbf{a}, \mathbf{0}, \mathbf{c}, \mathbf{h}, \mathbf{k}}^{\text {out }}\right)^{l}$, introduced in Section 3, are the structural coefficients of $\sum_{l=1}^{K}\left(f_{\mathbf{a}, \mathbf{0}, \mathbf{c}, \mathbf{h}, \mathbf{k}}^{\text {out }}\right)^{l} W_{\overline{\mathbf{a}}}^{l}$ that is the projection of $f_{\mathbf{a}, \mathbf{0}, \mathbf{c}, \mathbf{h}, \mathbf{k}}^{\text {out }}$ on the deficiency space $\mathcal{D}_{\mathbf{q}}(\overline{\mathbf{a}})$. The "tuning conditions" become hence

$$
\begin{aligned}
\tilde{a}_{l}^{2 m-2} & =\left(1+\frac{\left(f_{\mathbf{a}, \mathbf{0}, \mathbf{c}, \mathbf{h}, \mathbf{k}}^{\text {out }}\right)^{l}}{\varepsilon^{2 m-2}}\right) \bar{a}_{l}-\frac{k_{l}^{(0)} r_{\varepsilon}^{2 m-4}}{(4 m-8) e\left(\Gamma_{l}\right) \varepsilon^{2 m-2}} \\
C_{j} & =\frac{k_{j}^{(0)} \varepsilon^{2-2 m} r_{\varepsilon}^{2 m-4}}{4 m-8}
\end{aligned}
$$

Now these conditions allow us to proceed exactly as in [3, Subection 5.3], and the proof of 1.2 in the case $q \neq \emptyset$ is complete.

Let now $q=\emptyset$. Also in this case it is possible, using Propositions 4.4 and 4.10, to rescale, expand, and decompose the potentials $\mathcal{V}_{j, 0, \mathbf{b}, \mathbf{c}, \mathbf{h}, \mathbf{k}^{\prime}}^{\text {out }} \mathcal{V}_{j, \tilde{b}_{j}, \tilde{h}_{j}, \tilde{k}_{i}}^{\text {in }}$ The "tuning conditions", we 
must impose, are the following.

$$
\begin{aligned}
& c\left(\Gamma_{j}\right) \tilde{b}_{j}^{2 m} \varepsilon^{2} R_{\varepsilon}^{2-2 m}=\left(1-\frac{\left(f_{0, \mathbf{b}, \mathbf{c}, \mathbf{h}, \mathbf{k}}^{\text {out }}\right)^{j}}{\varepsilon^{2 m}}\right) c\left(\Gamma_{j}\right) B_{j}^{2 m} \varepsilon^{2 m} r_{\varepsilon}^{2-2 m}+\left(h_{j}^{(0)}+\frac{k_{j}^{(0)}}{4 m-8}\right) \\
& \quad-\left(1-\frac{\left(f_{0, \mathbf{b}, \mathbf{c}, \mathbf{h}, \mathbf{k}}^{\text {out }}\right)^{j}}{\varepsilon^{2 m}}\right) C_{j} \varepsilon^{2 m} r_{\varepsilon}^{4-2 m}-\frac{k_{j}^{(0)}}{4 m-8} \\
& =-\frac{c\left(\Gamma_{j}\right)\left(\mathrm{C}_{4, j}+\tilde{b}_{j} \varepsilon \mathrm{C}_{5, j}\right) \varepsilon^{4} \tilde{b}^{2 m} R_{\varepsilon}^{4-2 m}}{8(m-2)(m-1)}-\frac{c\left(\Gamma_{j}\right) \mathrm{c}_{0} \tilde{k}_{j}^{(0)} \tilde{b}_{j}^{2}}{32(m-2)(m-1) m R_{\varepsilon}^{2}} \\
& \quad-\frac{c\left(\Gamma_{j}\right) \mathrm{c}_{2} \tilde{b}_{j}^{2}}{(m-2)(m-1) R_{\varepsilon}^{2}}\left(\tilde{h}_{j}^{(2)}-\frac{\tilde{k}_{j}^{(2)}}{4(m+2)}\right)-\frac{c\left(\Gamma_{j}\right) \mathrm{c}_{3} \tilde{b}_{j}^{3}}{(m-2)(m-1) R_{\varepsilon}^{3}}\left(\tilde{h}_{j}^{(3)}-\frac{\tilde{k}_{j}^{(3)}}{4(m+3)}\right),
\end{aligned}
$$

where the quantities $\left(f_{0, \mathbf{b}, \mathbf{c}, \mathbf{h}, \mathbf{k}}^{\text {out }}\right)^{j}$, introduced in Section 3, are the structural coefficients of $\sum_{j=1}^{N}\left(f_{0, \mathbf{b}, \mathbf{c}, \mathbf{h}, \mathbf{k}}^{\text {out }}\right)^{l} W_{\mathbf{b}, \mathbf{c}}^{j}$ that is the projection of $f_{0, \mathbf{b}, \mathbf{c}, \mathbf{h}, \mathbf{k}}^{\text {out }}$ on the deficiency space $\mathcal{D}_{\mathbf{p}}(\mathbf{b}, \mathbf{c})$. First, we set

$$
\tilde{b}_{j}^{2 m}=B_{j}^{2 m}\left(1-\frac{\left(f_{0, \mathbf{b}, \mathbf{c}, \mathbf{h}, \mathbf{k}}^{\text {out }}\right)^{j}}{\varepsilon^{2 m}}\right)+\frac{1}{c\left(\Gamma_{j}\right)}\left(h_{j}^{(0)}+\frac{k_{j}^{(0)}}{4 m-8}\right) \frac{r_{\varepsilon}^{2 m-2}}{\varepsilon^{2 m}}
$$

then we solve equation (5.1) with respect to $C_{j}$ and hence we determine the remaining tuning parameters. Letting $\varepsilon$ tend to 0 we find, looking at the choices of tuning parameters, the correct relation intertwining coefficients $\mathbf{b}$ and $\mathbf{c}$ in the balancing condition that is

$$
c_{j}=b_{j}\left[\frac{1}{m} S_{\omega}\left(1+\frac{(m-1)^{2}}{(m+1)}\right)-\frac{c_{4, j}}{2(m-1)\left|\mathbb{S}^{2 m-1}\right|}\right]
$$

Now, the proof of Theorem 1.2 when $q=\emptyset$ follows arguing as in [2, Subsection 6.2]. Indeed, after observing that the terms containing $\mathrm{c}_{0, j}, \mathrm{C}_{2, j}, \mathrm{C}_{3, j}, \mathrm{C}_{5, j}$ have the $\varepsilon$-growth that is bigger than the $\varepsilon$-growth of pseudo-boundary data and hence those terms are dominated by the pseudo-boundary data, one can use the argument of data matching exposed in [2, Section 6].

\section{References}

[1] Arezzo, C. and F. Pacard. "Blowing up and desingularizing constant scalar curvature Kähler manifolds." Acta Mathematica 196, no. 2 (2006): 179-228. 
[2] Arezzo, C., R. Lena, and L. Mazzieri. “On the Kummer construction for kcsc metrics." (2015) http://arxiv.org/abs/1507.05105.

[3] Arezzo, C. and F. Pacard. "Blowing up Kähler manifolds with constant scalar curvature II." Annals of Mathematics (2) 170, no. 2 (2009): 685-738.

[4] Arezzo, C., F. Pacard, and M. Singer. "Extremal metrics on blowups." Duke Mathematical Journal 157, no. 1 (2011): 1-51.

[5] Bertin, J., J-P. Demailly, L. Illusie, and C. Peters. Introduction to Hodge Theory. SMF/AMS Texts and Monographs 8. Paris: Société Mathématique de France, 2002. Translated from the 1996 French original by James Lewis and Peters. Providence, RI: American Mathematical Society.

[6] Bochner, S. and W. T. Martin. Several Complex Variables. Princeton Mathematical Series 10. Princeton, NJ: Princeton University Press, 1948.

[7] Calabi, E. "Extremal Kähler Metrics. II." Differential Geometry and Complex Analysis, 95114. Berlin: Springer, 1985.

[8] Calderbank, D. M. J. and M. A. Singer. "Einstein metrics and complex singularities." Inventiones Mathematicae 156, no. 2 (2004): 405-43.

[9] Conlon, R. J. and H-J. Hein. “Asymptotically conical Calabi-Yau manifolds, I." Duke Mathematical Journal 162, no. 15 (2013): 2855-902.

[10] Goto, R. "Calabi-Yau structures and Einstein-Sasakian structures on crepant resolutions of isolated singularities." Journal of the Mathematical Society of Japan 64, no. 3 (2012): $1005-52$.

[11] Joyce, D. Compact Manifolds with Special Holonomy. Oxford Mathematical Monographs. Oxford: Oxford University Press, 2000.

[12] LeBrun, C. "Counter-examples to the generalized positive action conjecture." Communications in Mathematical Physics 118, no. 4 (1988): 591-6.

[13] LeBrun, C. "Explicit self-dual metrics on $\mathrm{CP}_{2} \# \cdots \# \mathrm{CP}_{2}$." Journal of Differential Geometry 34, no. 1 (1991): 223-53.

[14] LeBrun, C. and S. R. Simanca. "Extremal Kähler metrics and complex deformation theory." Geometric and Functional Analysis 4, no. 3 (1994): 298-336.

[15] McDuff, D. and D. Salamon. Introduction to Symplectic Topology. Oxford Mathematical Monographs. 2nd ed. New York: The Clarendon Press, Oxford University Press, 1998.

[16] Rollin, Y. and M. Singer. “Constant scalar curvature Kähler surfaces and parabolic polystability." The Journal of Geometric Analysis 19, no. 1 (2009): 107-36.

[17] Stoppa, J. "Unstable blowups." Journal of Algebraic Geometry 19, no. 1 (2010): 1-17.

[18] Székelyhidi, G. "On blowing up extremal Kähler manifolds." Duke Mathematical Journal 161, no. 8 (2012): 1411-53.

[19] Tipler, C. "Extremal Kähler metrics on blow-ups of parabolic ruled surfaces." Bulletin de la Société Mathématique de France 141, no. 3 (2013): 481-516.

[20] van Coevering, C. "Ricci-flat Kähler metrics on crepant resolutions of Kähler cones." Mathematische Annalen 347, no. 3 (2010): 581-611. 
38 Claudio Arezzo et al.

[21] Vedova, A. D. and F. Zuddas. "Scalar curvature and asymptotic Chow stability of projective bundles and blowups." Transactions of the American Mathematical Society 364, no. 12 (2012): 6495-511.

[22] Verbitsky, M. "Holomorphic symplectic geometry and orbifold singularities." The Asian Journal of Mathematics 4, no. 3 (2000): 553-63.

[23] Viaclovsky, J. and M. T. Lock. "A Smörgåsbord of scalar-flat Kähler ALE surfaces." (2014): preprint http://arxiv.org/abs/1410.6461. 